J. DIFFERENTIAL GEOMETRY

92 (2012) 507-545

\title{
THE SPHERE THEOREMS FOR MANIFOLDS WITH POSITIVE SCALAR CURVATURE
}

\author{
JuAn-Ru Gu \& Hong-Wei Xu
}

\begin{abstract}
Some new differentiable sphere theorems are obtained via the Ricci flow and stable currents. We prove that if $M^{n}$ is a compact manifold whose normalized scalar curvature and sectional curvature satisfy the pointwise pinching condition $R_{0}>\sigma_{n} K_{\max }$, where $\sigma_{n} \in\left(\frac{1}{4}, 1\right)$ is an explicit positive constant, then $M$ is diffeomorphic to a spherical space form. We also provide a partial answer to Yau's conjecture on the pinching theorem. Moreover, we prove that if $M^{n}(n \geq 3)$ is a compact manifold whose $(n-2)$-th Ricci curvature and normalized scalar curvature satisfy the pointwise condition $\operatorname{Ric}_{\min }^{(n-2)}>\tau_{n}(n-2) R_{0}$, where $\tau_{n} \in\left(\frac{1}{4}, 1\right)$ is an explicit positive constant, then $M$ is diffeomorphic to a spherical space form. We then extend the sphere theorems above to submanifolds in a Riemannian manifold. Finally we give a classification of submanifolds with weakly pinched curvatures, which improves the differentiable pinching theorems due to Andrews, Baker, and the authors.
\end{abstract}

\section{Introduction}

Studying curvature and topology of manifolds plays an important role in global differential geometry. The sphere theorem for Riemannian manifolds was initiated by Rauch [37] in 1951. During the past six decades, there has been much progress on sphere theorems for Riemannian manifolds and submanifolds $[\mathbf{3}, \mathbf{6}, \mathbf{8}, \mathbf{1 1}, \mathbf{4 3}, 44,49]$. The Brendle-Schoen Differentiable Sphere Theorem $[\mathbf{9}, \mathbf{1 0}]$ brought us a big breakthrough in the investigation of curvature and topology of manifolds. The following results, due to Brendle and Schoen $[\mathbf{5}, \mathbf{1 0}]$, are very important throughout this paper.

Theorem A ([5]). Let $\left(M, g_{0}\right)$ be a compact Riemannian manifold of dimension $n(\geq 4)$. Assume that

$$
R_{1313}+\lambda^{2} R_{1414}+R_{2323}+\lambda^{2} R_{2424}-2 \lambda R_{1234}>0
$$

Received 2/10/2011. 
for all orthonormal four-frames $\left\{e_{1}, e_{2}, e_{3}, e_{4}\right\}$ and all $\lambda \in[0,1]$. Then the normalized Ricci flow with initial metric $g_{0}$,

$$
\frac{\partial}{\partial t} g(t)=-2 R i c_{g(t)}+\frac{2}{n} r_{g(t)} g(t)
$$

exists for all time and converges to a constant curvature metric as $t \rightarrow$ $\infty$. Here $r_{g(t)}$ denotes the mean value of the scalar curvature of $g(t)$.

Theorem B ([10]). Let $\left(M, g_{0}\right)$ be a compact, locally irreducible Riemannian manifold of dimension $n(\geq 4)$. Assume that $M \times \mathbb{R}^{2}$ has nonnegative isotropic curvature, i.e.,

$$
R_{1313}+\lambda^{2} R_{1414}+\mu^{2} R_{2323}+\lambda^{2} \mu^{2} R_{2424}-2 \lambda \mu R_{1234} \geq 0
$$

for all orthonormal four-frames $\left\{e_{1}, e_{2}, e_{3}, e_{4}\right\}$ and all $\lambda, \mu \in[-1,1]$. Then one of the following statements holds:

(i) $M$ is diffeomorphic to a spherical space form.

(ii) $n=2 m$ and the universal cover of $M$ is a Kähler manifold biholomorphic to $\mathbb{C} P^{m}$.

(iii) The universal cover of $M$ is isometric to a compact symmetric space.

On the other hand, some important work on sphere theorems for manifolds with positive Ricci curvature has been done by several geometers (see $[\mathbf{3}, \mathbf{1 5}, \mathbf{2 3}, \mathbf{3 2}, \mathbf{3 4}, \mathbf{4 0}, \mathbf{4 2}, \mathbf{4 4}]$, etc.). In the 1990s, Cheeger, Colding, and Petersen $[\mathbf{1 5}, \mathbf{3 4}]$ proved the following differentiable sphere theorem for manifolds with positive Ricci curvature.

Theorem C ([15, 34]). Let $M^{n}$ be a compact Riemannian n-manifold with Ricci curvature Ric $_{M} \geq n-1$. Suppose that one of the following conditions holds:

(i) $\operatorname{vol}(M)>\omega_{n}-\varepsilon_{n}$, where $\omega_{n}=\operatorname{vol}\left(S^{n}\right)$ and $\varepsilon_{n}$ is some positive constant;

(ii) $\lambda_{n+1}<n+\theta_{n}$, where $\lambda_{n+1}$ is the $(n+1)$-th eigenvalue of $M$ and $\theta_{n}$ is some positive constant.

Then $M$ is diffeomorphic to $S^{n}$.

Let $K(\pi)$ be the sectional curvature of $M$ for 2-plane $\pi \subset T_{x} M$, and $\operatorname{Ric}(u)$ the Ricci curvature of $M$ for unit vector $u \in U_{x} M$. Set $K_{\max }(x):=\max _{\pi \subset T_{x} M} K(\pi), \operatorname{Ric}_{\min }(x):=\min _{u \in U_{x} M} \operatorname{Ric}(u)$. Inspired by Shen's topological sphere theorem $[\mathbf{4 0}]$, the authors $[\mathbf{5 0}]$ obtained the following differentiable sphere theorem for manifolds of positive Ricci curvatures.

Theorem D ([50]). Let $M^{n}$ be a compact Riemannian $n$-manifold $(n \geq 3)$. If Ric $c_{\min }>\delta_{n}(n-1) K_{\max }$, where $\delta_{n}=1-\frac{6}{5(n-1)}$, then $M$ is diffeomorphic to a spherical space form. In particular, if $M$ is simply connected, then $M$ is diffeomorphic to $S^{n}$. 
Let $M^{n}$ be a submanifold in a Riemannian manifold $\bar{M}^{N}$. Denote by $H$ and $S$ the mean curvature and the squared length of the second fundamental form of $M$, respectively. Denote by $\bar{K}(\pi)$ the sectional curvature of $\bar{M}$ for 2-plane $\pi\left(\subset T_{x} \bar{M}\right)$. Set $\bar{K}_{\max }(x):=\max _{\pi \subset T_{x}} \bar{M} \bar{K}(\pi)$, $\bar{K}_{\min }(x):=\min _{\pi \subset T_{x}} \bar{M} \bar{K}(\pi)$. In [53], Xu and Zhao obtained some differentiable sphere theorems for complete submanifolds in higher codimensions via the Ricci flow and stable currents. Recently the authors [51] proved the following differentiable sphere theorem for complete submanifolds with strictly pinched curvatures, which is a generalization of the Brendle-Schoen sphere theorem [9].

Theorem E ([51]). Let $M^{n}$ be an $n$-dimensional complete submanifold in an $N$-dimensional Riemannian manifold $\bar{M}^{N}$. If $S<\frac{8}{3}\left(\bar{K}_{\text {min }}-\right.$ $\left.\frac{1}{4} \bar{K}_{\max }\right)+\frac{n^{2} H^{2}}{n-1}$, then $M$ is diffeomorphic to a spherical space form or $\mathbb{R}^{n}$. In particular, if $M$ is simply connected, then $M$ is diffeomorphic to $S^{n}$ or $\mathbb{R}^{n}$.

The purpose of this paper is to prove some new differentiable sphere theorems for Riemannian manifolds and submanifolds. In Section 3, we prove the following differentiable sphere theorem for compact manifolds with positive scalar curvature.

Theorem 1. Let $M^{n}$ be an $n(\geq 3)$-dimensional compact Riemannian manifold. Denote by $R_{0}$ the normalized scalar curvature of $M$. Assume that one of the following pointwise pinching conditions holds:

(i) $R_{0}>\sigma_{n} K_{\max }$;

(ii) $K_{\min }>\eta_{n} R_{0}$.

Then $M$ is diffeomorphic to a spherical space form. In particular, if $M$ is simply connected, then $M$ is diffeomorphic to $S^{n}$. Here

$$
\begin{aligned}
& \sigma_{n}= \begin{cases}1-\frac{4}{n(n-1)} & \text { for } n=3, \\
1-\frac{12}{5 n(n-1)} & \text { for } n \geq 4,\end{cases} \\
& \eta_{n}=1-\frac{6}{n^{2}-n+6} .
\end{aligned}
$$

Theorem 1 improves Theorem D and gives a partial answer to Yau's conjecture on the pointwise pinching theorem (see [54], Problem 12). Moreover, we obtain the following theorem.

Theorem 2. Let $M^{n}$ be an $n(\geq 3)$-dimensional compact Riemannian manifold. Denote by $R_{0}$ and Ric ${ }^{(n-2)}$ the normalized scalar curvature and the (n-2)-th Ricci curvature of $M$. Assume that one of the following pointwise pinching conditions holds:

(i) $(n-2) R_{0}>\mu_{n} \operatorname{Ric}_{\max }^{(n-2)}$;

(ii) $\operatorname{Ric}_{\min }^{(n-2)}>\tau_{n}(n-2) R_{0}$. 
Then $M$ is diffeomorphic to a spherical space form. In particular, if $M$ is simply connected, then $M$ is diffeomorphic to $S^{n}$. Here

$$
\begin{aligned}
& \mu_{n}=1-\frac{6}{n(n-1)(n+1)}, \\
& \tau_{n}=\max \left\{1-\frac{12}{(n-2)\left(5 n^{2}-11 n-6\right)}, 0\right\} .
\end{aligned}
$$

REMARK 1 . We consider the case of $n \geq 4$. Note that differentiable structures of Einstein manifolds are very rich. If pinching conditions (i) and (ii) in Theorem 2 are replaced by $(n-1) R_{0}>\tilde{\mu}_{n} R i c_{\max }$ and $R i c_{\min }>\tilde{\tau}_{n}(n-1) R_{0}$ respectively, where $\tilde{\mu}_{n}, \tilde{\tau}_{n} \in\left(\frac{1}{4}, 1\right)$, it's impossible to obtain the same assertion. Therefore, the pinching conditions in Theorem 2 are the weakest in this sense.

In Section 4, we extend the sphere theorems above to submanifolds in a Riemannian manifold with arbitrary codimension (Theorems 13 and 14). In Section 5, we obtain a differentiable sphere theorem for submanifolds with weakly pinched curvatures, stated as:

Theorem 3. Let $M^{n}$ be an $n(\geq 3)$-dimensional compact submanifold in an N-dimensional Riemannian manifold $\bar{M}^{N}$. Assume that $M$ satisfies one of the following conditions:

(i) $\bar{K}_{\min }\left(x_{0}\right)-\frac{1}{4} \bar{K}_{\max }\left(x_{0}\right) \neq 0$ for some point $x_{0} \in M$, and $S \leq$ $\frac{8}{3}\left(\bar{K}_{\min }-\frac{1}{4} \bar{K}_{\max }\right)+\frac{n^{2} H^{2}}{n-1}$;

(ii) $\bar{K}_{\min }(x)-\frac{1}{4} \bar{K}_{\max }(x)=0$ for any point $x \in M, S \leq \frac{n^{2} H^{2}}{n-1}$, and the strict inequality holds for some point $x_{0} \in M$.

Then $M$ is diffeomorphic to a spherical space form. In particular, if $M$ is simply connected, then $M$ is diffeomorphic to $S^{n}$.

Furthermore, we prove the following classification theorem of submanifolds with weakly pinched curvatures in space forms.

Theorem 4. Let $M^{n}$ be an $n(\geq 4)$-dimensional oriented complete submanifold in an $N$-dimensional simply connected space form $F^{N}(c)$ with $c \geq 0$. Assume that its scalar curvature $R \geq(n+1)(n-2) c+$ $\frac{n^{2}(n-2)}{n-1} H^{2}$, where $c+H^{2}>0$. We have

(i) If $c=0$, then $M$ is either diffeomorphic to $S^{n}, \mathbb{R}^{n}$, or locally isometric to $S^{n-1}(r) \times \mathbb{R}$.

(ii) If $M$ is compact, then $M$ is diffeomorphic to $S^{n}$.

REMARK 2. The pinching condition in Theorem 4 is equivalent to $S \leq 2 c+\frac{n^{2} H^{2}}{n-1}$. Theorems 3 and 4 improve the differentiable pinching theorems due to Andrews-Baker and the authors [1, 51].

It should be mentioned that the second author introduced the results above in his invited talk at the Fifth International Congress of Chinese 
Mathematicians held in Beijing from December 17 to December 22, 2010 .

Acknowledgments. The authors would like to thank Professors Kefeng Liu, Mu-Tao Wang, Shing-Tung Yau, and Fangyang Zheng for their helpful discussions and valuable suggestions.

This research was supported by the NSFC, Grant Nos. 11071211, 10771187; and the Trans-Century Training Programme Foundation for Talents by the Ministry of Education of China.

\section{Notation and lemmas}

Let $M^{n}$ be an $n$-dimensional submanifold in an $N$-dimensional Riemannian manifold $\bar{M}^{N}$. We shall make use of the following convention on the range of indices.

$$
\begin{aligned}
& 1 \leq A, B, C, \ldots \leq N ; 1 \leq i, j, k, \ldots \leq n ; \\
& \text { if } N \geq n+1, n+1 \leq \alpha, \beta, \gamma, \ldots \leq N .
\end{aligned}
$$

For an arbitrary fixed point $x \in M \subset \bar{M}$, we choose an orthonormal local frame field $\left\{e_{A}\right\}$ in $\bar{M}^{N}$ such that $e_{i}$ 's are tangent to $M$. Denote by $\left\{\omega_{A}\right\}$ the dual frame field of $\left\{e_{A}\right\}$. Let

$$
\begin{aligned}
& R m=\sum_{i, j, k, l} R_{i j k l} \omega_{i} \otimes \omega_{j} \otimes \omega_{k} \otimes \omega_{l}, \\
& \overline{R m}=\sum_{A, B, C, D} \bar{R}_{A B C D} \omega_{A} \otimes \omega_{B} \otimes \omega_{C} \otimes \omega_{D}
\end{aligned}
$$

be the Riemannian curvature tensors of $M$ and $\bar{M}$, respectively. Denote by $h$ and $\xi$ the second fundamental form and the mean curvature vector of $M$. When $N=n, h$ and $\xi$ are identically equal to zero. When $N \geq$ $n+1$, we set

$$
h=\sum_{\alpha, i, j} h_{i j}^{\alpha} \omega_{i} \otimes \omega_{j} \otimes e_{\alpha}, \quad \xi=\frac{1}{n} \sum_{\alpha, i} h_{i i}^{\alpha} e_{\alpha} .
$$

The squared norm $S$ of the second fundamental form and the mean curvature $H$ of $M$ are given by $S:=\sum_{\alpha, i, j}\left(h_{i j}^{\alpha}\right)^{2}, H:=|\xi|$. Then we have the Gauss equation

$$
R_{i j k l}=\bar{R}_{i j k l}+\left\langle h\left(e_{i}, e_{k}\right), h\left(e_{j}, e_{l}\right)\right\rangle-\left\langle h\left(e_{i}, e_{l}\right), h\left(e_{j}, e_{k}\right)\right\rangle .
$$

Denote by $K(\cdot), \bar{K}(\cdot), \operatorname{Ric}(\cdot), \overline{\operatorname{Ric}}(\cdot), R$, and $\bar{R}$ the sectional curvatures, the Ricci curvatures, and the scalar curvatures of $M$ and $\bar{M}$, respectively. Then we have

$$
\operatorname{Ric}\left(e_{i}\right)=\sum_{j} R_{i j i j}, \overline{\operatorname{Ric}}\left(e_{A}\right)=\sum_{B} \bar{R}_{A B A B}
$$




$$
R=\sum_{i, j} R_{i j i j}, \quad \bar{R}=\sum_{A, B} \bar{R}_{A B A B}
$$

Set $K_{\min }(x)=\min _{\pi \subset T_{x} M} K(\pi), K_{\max }(x)=\max _{\pi \subset T_{x} M} K(\pi), \bar{K}_{\min }(x)=$ $\min _{\pi \subset T_{x}} \bar{M} \bar{K}(\pi), \bar{K}_{\max }(x)=\max _{\pi \subset T_{x}} \bar{M} \bar{K}(\pi)$. Then by Berger's inequality (see e.g. [6], Proposition 1.9), we have

$$
\left|R_{i j k l}\right| \leq \frac{2}{3}\left(K_{\max }-K_{\min }\right)
$$

for all distinct indices $i, j, k, l$, and

$$
\left|\bar{R}_{A B C D}\right| \leq \frac{2}{3}\left(\bar{K}_{\max }-\bar{K}_{\min }\right)
$$

for all distinct indices $A, B, C, D$. We set

$$
\begin{aligned}
& R i c_{\min }(x)=\min _{u \in U_{x} M} \operatorname{Ric}(u), \overline{\operatorname{Ric}}_{\min }(x)=\min _{u \in U_{x} \bar{M}} \overline{\operatorname{Ric}}(u), \\
& R i c_{\max }(x)=\max _{u \in U_{x} M} \operatorname{Ric}(u), \overline{\operatorname{Ric}}_{\max }(x)=\max _{u \in U_{x} \bar{M}} \overline{\operatorname{Ric}}(u) .
\end{aligned}
$$

For any unit tangent vector $u \in U_{x} M$ at point $x \in M$, let $V_{x}^{k}$ be a $k$ dimensional subspace of $T_{x} M$ satisfying $u \perp V_{x}^{k}$. Choose an orthonormal basis $\left\{e_{i}\right\}$ in $T_{x} M$ such that $e_{j_{0}}=u, \operatorname{span}\left\{e_{j_{1}}, \ldots, e_{j_{k}}\right\}=V_{x}^{k}$, where the indices $1 \leq j_{0}, j_{1}, \ldots, j_{k} \leq n$ are distinct with each other. We set

$$
\begin{aligned}
& \operatorname{Ric}^{(k)}\left(u ; V_{x}^{k}\right)=\operatorname{Ric}^{(k)}\left(\left[e_{j_{0}}, \ldots, e_{j_{k}}\right]\right)=\sum_{q=1}^{k} R_{j_{0} j_{q} j_{0} j_{q}}, \\
& \operatorname{Ric}_{\min }^{(k)}(x)=\min _{u \in U_{x} M} \min _{u \perp V_{x}^{k} \subset T_{x} M} \operatorname{Ric}^{(k)}\left(u ; V_{x}^{k}\right), \\
& \operatorname{Ric}_{\max }^{(k)}(x)=\max _{u \in U_{x} M} \max _{u \perp V_{x}^{k} \subset T_{x} M} \operatorname{Ric}^{(k)}\left(u ; V_{x}^{k}\right) .
\end{aligned}
$$

We extend an orthonormal $s$-frame $\left\{e_{j_{0}}, \ldots, e_{j_{s-1}}\right\}$ in $T_{x} M$ to $(k+1)$ frame $\left\{e_{j_{0}}, \ldots, e_{j_{k}}\right\}$ for $1 \leq s \leq k+1 \leq n$ and set

$$
\begin{aligned}
& R^{(k, s)}\left(\left[e_{j_{0}}, \ldots, e_{j_{k}}\right]\right)=\sum_{p=0}^{s-1} \sum_{q=0}^{k} R_{j_{p} j_{q} j_{p} j_{q}}, \\
& R_{\min }^{(k, s)}(x)=\min _{\left\{e_{j_{0}}, \ldots, e_{j_{k}}\right\} \subset T_{x} M} R^{(k, s)}\left(\left[e_{j_{0}}, \ldots, e_{j_{k}}\right]\right), \\
& R_{\max }^{(k, s)}(x)=\max _{\left\{e_{j_{0}}, \ldots, e_{j_{k}}\right\} \subset T_{x} M} R^{(k, s)}\left(\left[e_{j_{0}}, \ldots, e_{j_{k}}\right]\right) .
\end{aligned}
$$




$$
\begin{aligned}
& \operatorname{Ric}^{[s]}\left(\left[e_{j_{0}}, \ldots, e_{j_{n-1}}\right]\right)=R^{(n-1, s)}\left(\left[e_{j_{0}}, \ldots, e_{j_{n-1}}\right]\right) \\
& =\sum_{p=0}^{s-1} \sum_{q=0}^{n-1} R_{j_{p} j_{q} j_{p} j_{q}}, \\
& \operatorname{Ric}_{\min }^{[s]}(x)=\min _{\left\{e_{j_{0}}, \ldots, e_{j_{n-1}}\right\} \subset T_{x} M} \operatorname{Ric}^{[s]}\left(\left[e_{j_{0}}, \ldots, e_{j_{n-1}}\right]\right) \text {, } \\
& \operatorname{Ric}_{\max }^{[s]}(x)=\max _{\left\{e_{j_{0}}, \ldots, e_{j_{n-1}}\right\} \subset T_{x} M} \operatorname{Ric}^{[s]}\left(\left[e_{j_{0}}, \ldots, e_{j_{n-1}}\right]\right) . \\
& R^{(k)}\left(\left[e_{j_{0}}, \ldots, e_{j_{k}}\right]\right)=R^{(k, k+1)}\left(\left[e_{j_{0}}, \ldots, e_{j_{k}}\right]\right) \\
& =\sum_{p=0}^{k} \sum_{q=0}^{k} R_{j_{p} j_{q} j_{p} j_{q}}, \\
& R_{\text {min }}^{(k)}(x)=\min _{\left\{e_{j_{0}}, \ldots, e_{j_{k}}\right\} \subset T_{x} M} R^{(k)}\left(\left[e_{j_{0}}, \ldots, e_{j_{k}}\right]\right), \\
& R_{\max }^{(k)}(x)=\max _{\left\{e_{j_{0}}, \ldots, e_{j_{k}}\right\} \subset T_{x} M} R^{(k)}\left(\left[e_{j_{0}}, \ldots, e_{j_{k}}\right]\right) .
\end{aligned}
$$

Definition 1. We call $\operatorname{Ric}^{(k)}\left(u ; V_{x}^{k}\right), \quad R^{(k, s)}\left(\left[e_{j_{0}}, \ldots, e_{j_{k}}\right]\right)$, $\operatorname{Ric}^{[s]}\left(\left[e_{j_{0}}, \ldots, e_{j_{n-1}}\right]\right)$, and $R^{(k)}\left(\left[e_{j_{0}}, \ldots, e_{j_{k}}\right]\right)$ the $k$-th Ricci curvature, $(k, s)$-curvature, $s$-th weak Ricci curvature, and $k$-th scalar curvature of $M$, respectively.

The geometry and topology of $k$-th Ricci curvature was initiated by Hartman $[\mathbf{2 2}]$ in 1979, and developed by $\mathrm{Wu}[\mathbf{4 7}]$ and Shen $[\mathbf{4 0 , 4 1}$, etc. By the definition above, it is seen that the Ricci curvature of $M$ is equal to the $(n-1)$-th Ricci curvature, $(n-1,1)$-curvature, and 1 -th weak Ricci curvature; the scalar curvature of $M$ is equal to $(n-1, n)$ curvature, $n$-th weak Ricci curvature, and $(n-1)$-th scalar curvature. For any unit tangent vector $u \in U_{x} \bar{M}$ at point $x \in \bar{M}$, let $V_{x}^{k}$ be a $k$ dimensional subspace of $T_{x} \bar{M}$ satisfying $u \perp V_{x}^{k}$. Choose an orthonormal basis $\left\{e_{A}\right\}$ in $T_{x} \bar{M}$ such that $e_{A_{0}}=u, \operatorname{span}\left\{e_{A_{1}}, \ldots, e_{A_{k}}\right\}=V_{x}^{k}$, where the indices $1 \leq A_{0}, A_{1}, \ldots, A_{k} \leq N$ are distinct with each other. We define the $k$-th Ricci curvature as follows:

$$
\overline{\operatorname{Ric}}^{(k)}\left(u ; V_{x}^{k}\right)=\sum_{q=1}^{k} \bar{R}_{A_{0} A_{q} A_{0} A_{q}} .
$$

We extend an orthonormal $s$-frame $\left\{e_{A_{0}}, \ldots, e_{A_{s-1}}\right\}$ in $T_{x} \bar{M}$ to $(k+1)$ frame $\left\{e_{A_{0}}, \ldots, e_{A_{k}}\right\}$ for $1 \leq s \leq k+1 \leq N$ and define the $(k, s)$ curvature, $s$-th weak Ricci curvature, and $k$-th scalar curvature of $\bar{M}$ as 
follows:

$$
\begin{aligned}
\bar{R}^{(k, s)}\left(\left[e_{A_{0}}, \ldots, e_{A_{k}}\right]\right) & =\sum_{p=0}^{s-1} \sum_{q=0}^{k} \bar{R}_{A_{p} A_{q} A_{p} A_{q}}, \\
\overline{\operatorname{Ric}}^{[s]}\left(\left[e_{A_{0}}, \ldots, e_{A_{N-1}}\right]\right) & =\bar{R}^{(N-1, s)}\left(\left[e_{A_{0}}, \ldots, e_{A_{N-1}}\right]\right) \\
& =\sum_{p=0}^{s-1} \sum_{q=0}^{N-1} \bar{R}_{A_{p} A_{q} A_{p} A_{q}}, \\
\bar{R}^{(k)}\left(\left[e_{A_{0}}, \ldots, e_{A_{k}}\right]\right)= & \bar{R}^{(k, k+1)}\left(\left[e_{A_{0}}, \ldots, e_{A_{k}}\right]\right) \\
= & \sum_{p=0}^{k} \sum_{q=0}^{k} \bar{R}_{A_{p} A_{q} A_{p} A_{q} .} .
\end{aligned}
$$

Denote by $\overline{R i c}_{\min }^{(k)}(x), \bar{R}_{\min }^{(k, s)}(x), \overline{R i c}_{\min }^{[s]}(x), \bar{R}_{\min }^{(k)}(x)$ and $\overline{R i c}_{\max }^{(k)}(x)$, $\bar{R}_{\max }^{(k, s)}(x), \overline{R i c}_{\max }^{[s]}(x), \bar{R}_{\max }^{(k)}(x)$ the minimum and maximum of the curvatures defined above at point $x \in \bar{M}$.

We choose an orthonormal frame $\left\{e_{1}, e_{2}, \cdots, e_{n}\right\}$ such that $u=e_{n}$ and $\operatorname{Ric}^{(k)}\left(u ; V_{x}^{k}\right)=\sum_{i=1}^{k} R_{\text {inin }}$, where $V_{x}^{k}=\operatorname{span}\left\{e_{1}, e_{2}, \cdots, e_{k}\right\}, 1 \leq$ $k \leq n-1$. In particular, we see that $\operatorname{Ric}^{(n-1)}\left(u ; V_{x}^{n-1}\right)=\operatorname{Ric}(u)$ and $\operatorname{Ric}^{(1)}\left(u ; V_{x}^{1}\right)=K(\pi)$, where $\pi=\operatorname{span}\left\{e_{1}, e_{n}\right\}$. Then we have the following lemma.

Lemma 1. Let $M^{n}$ be an $n$-dimensional complete submanifold in an $N$-dimensional Euclidean space $\mathbb{R}^{N}$. If $S \leq \frac{n^{2} H^{2}}{n-1}, H \neq 0$, then

(i) $([\mathbf{4 5}, \mathbf{5 1}]) \operatorname{Ric}^{(k)}\left(u ; V_{x}^{k}\right) \geq 0$.

(ii) For each point $x \in M$ there exists a unit vector $u$ such that $\operatorname{Ric}^{(k)}\left(u ; V_{x}^{k}\right)=0$ for some integer $k \in[2, n-1]$ if and only if $H$ is a constant and $M$ is isometric to $S^{n-1}\left(\frac{n-1}{n H}\right) \times \mathbb{R}$.

Proof. If $k=1$, the assertion follows from the result in [51]. Now we discuss the case for $2 \leq k \leq n-1$. Choose an orthonormal frame $\left\{e_{1}, e_{2}, \cdots, e_{N}\right\}$ such that $e_{n+1}$ is parallel to the mean curvature vector $\xi$. Then

$$
\begin{aligned}
n^{2} H^{2}= & \left(\sum_{i=1}^{n} h_{i i}^{n+1}\right)^{2} \\
= & (n-1)\left[\sum_{i=1}^{n}\left(h_{i i}^{n+1}\right)^{2}+\sum_{i \neq j}\left(h_{i j}^{n+1}\right)^{2}+\sum_{\alpha=n+2}^{N} \sum_{i, j=1}^{n}\left(h_{i j}^{\alpha}\right)^{2}\right. \\
& \left.+\frac{n^{2} H^{2}}{n-1}-S\right] .
\end{aligned}
$$


Note that for $l \neq n$

$$
\begin{aligned}
\left(\sum_{i=1}^{n} h_{i i}^{n+1}\right)^{2} & \leq(n-1)\left[\left(h_{l l}^{n+1}+h_{n n}^{n+1}\right)^{2}+\sum_{i \neq l, n}\left(h_{i i}^{n+1}\right)^{2}\right] \\
& =(n-1)\left[\sum_{i=1}^{n}\left(h_{i i}^{n+1}\right)^{2}+2 h_{l l}^{n+1} h_{n n}^{n+1}\right] .
\end{aligned}
$$

This together with (2.10) implies

$$
2 h_{l l}^{n+1} h_{n n}^{n+1} \geq \sum_{i \neq j}\left(h_{i j}^{n+1}\right)^{2}+\sum_{\alpha=n+2}^{N} \sum_{i, j=1}^{n}\left(h_{i j}^{\alpha}\right)^{2}+\frac{n^{2} H^{2}}{n-1}-S
$$

for $l \neq n$. The equality holds if and only if $h_{i i}^{n+1}=h_{l l}^{n+1}+h_{n n}^{n+1}$ for $i \neq l, n$. This together with (2.1) implies

$$
\begin{aligned}
\operatorname{Ric}^{(k)}\left(u ; V_{x}^{k}\right)= & \sum_{i=1}^{k} R_{\text {inin }}=\sum_{i=1}^{k} \sum_{\alpha=n+1}^{N}\left[h_{i i}^{\alpha} h_{n n}^{\alpha}-\left(h_{i n}^{\alpha}\right)^{2}\right] \\
\geq & k \sum_{\alpha=n+1}^{N} \sum_{1 \leq i<j<n}\left(h_{i j}^{\alpha}\right)^{2}+(k-1) \sum_{\alpha=n+1}^{N} \sum_{i=1}^{n-1}\left(h_{i n}^{\alpha}\right)^{2} \\
& +\frac{k-1}{2} \sum_{\alpha=n+2}^{N} \sum_{i=1}^{n-1}\left(h_{i i}^{\alpha}\right)^{2}+\frac{1}{2} \sum_{\alpha=n+2}^{N} \sum_{i=1}^{k}\left(h_{i i}^{\alpha}+h_{n n}^{\alpha}\right)^{2} \\
& +\frac{k}{2}\left(\frac{n^{2} H^{2}}{n-1}-S\right) \\
\geq & \frac{k}{2}\left(\frac{n^{2} H^{2}}{n-1}-S\right) .
\end{aligned}
$$

If $S \leq \frac{n^{2} H^{2}}{n-1}$, then $\operatorname{Ric}^{(k)}\left(u ; V_{x}^{k}\right) \geq 0$. The equality holds if and only if

$$
\begin{gathered}
h_{i j}^{\alpha}=0,1 \leq i, j \leq n, \alpha \neq n+1 ; h_{i j}^{n+1}=0, i \neq j, 1 \leq i, j \leq n ; \\
h_{n n}^{n+1}=0 ; \quad h_{i i}^{n+1}=\frac{n H}{n-1}, 1 \leq i \leq n-1 .
\end{gathered}
$$

Hence $M$ has essential codimension one. Since the shape operator of $M$ has one eigenvalue of multiplicity $n-1$ and the other eigenvalue is zero, it follows from a result due to Deprez (see [16], Corollary) that $H$ is a constant and $M$ is isometric to $S^{n-1}\left(\frac{n-1}{n H}\right) \times \mathbb{R}$. This completes the proof.

q.e.d.

The following nonexistence theorem for stable currents in a compact Riemannian manifold $M$ isometrically immersed into $F^{N}(c)$ is employed to eliminate the homology groups $H_{q}(M ; \mathbb{Z})$ for $0<q<n$, which was initiated by Lawson-Simons [28] and extended by Xin [48]. 
Theorem 5. Let $M^{n}$ be a compact submanifold in $F^{N}(c)$ with $c \geq 0$. Assume that

$$
\sum_{k=q+1}^{n} \sum_{i=1}^{q}\left[2\left|h\left(e_{i}, e_{k}\right)\right|^{2}-\left\langle h\left(e_{i}, e_{i}\right), h\left(e_{k}, e_{k}\right)\right\rangle\right]<q(n-q) c
$$

holds for any orthonormal basis $\left\{e_{i}\right\}$ of $T_{x} M$ at any point $x \in M$, where $q$ is an integer satisfying $0<q<n$. Then there do not exist any stable q-currents. Moreover,

$$
H_{q}(M ; \mathbb{Z})=H_{n-q}(M ; \mathbb{Z})=0,
$$

where $H_{i}(M ; \mathbb{Z})$ is the $i$-th homology group of $M$ with integer coefficients, and $\pi_{1}(M)=0$ when $q=1$.

From the proof of Lemma 2 in [45], we have

$$
\begin{aligned}
& \sum_{k=q+1}^{n} \sum_{i=1}^{q}\left[2\left|h\left(e_{i}, e_{k}\right)\right|^{2}-\left\langle h\left(e_{i}, e_{i}\right), h\left(e_{k}, e_{k}\right)\right\rangle\right] \\
\leq & \frac{q(n-q)}{n}\left[S-2 n H^{2}+\frac{\sqrt{n}|2 q-n|}{\sqrt{q(n-q)}} H \sqrt{S-n H^{2}}\right] \\
\leq & \frac{q(n-q)}{n}\left[S-2 n H^{2}+\frac{n(n-4)}{\sqrt{2 n(n-2)}} H \sqrt{S-n H^{2}}\right],
\end{aligned}
$$

for $n \geq 4$ and $1<q<n-1$. This together with Theorem 5 implies the following.

Lemma 2. Let $M^{n}$ be an $n(\geq 4)$-dimensional compact submanifold in a Euclidean space $\mathbb{R}^{N}$. If $S<\frac{n^{2} H^{2}}{n-2}$, then

$$
H_{q}(M ; \mathbb{Z})=0, \text { for all } 1<q<n-1 \text {. }
$$

Lemma 3 ([21]). Let $M$ be a compact Riemannian manifold of dimension $n$. If $M$ has nonnegative isotropic curvature and has positive isotropic curvature for some point in $M$, then $M$ admits a metric with positive isotropic curvature.

Lemma 4. Let $M$ be a compact Riemannian manifold of dimension $n$. If $M \times \mathbb{R}^{2}$ has nonnegative isotropic curvature, and if $M$ has positive Ricci curvature and isotropic curvature, then $M$ is diffeomorphic to a spherical space form.

Proof. By the assumption that $M$ has positive Ricci curvature, the universal cover $\widetilde{M}$ of $M$ is compact. Since $M$ has positive isotropic curvature, $\widetilde{M}$ also has positive isotropic curvature. Note that $\widetilde{M}$ is simply connected. It follows from a theorem due to Micallef and Moore [29] that $\widetilde{M}$ is homeomorphic to $S^{n}$. Therefore, $M$ is locally irreducible and the symmetric metric of $\widetilde{M}$ would have to be of positive constant curvature. Moreover, when $n$ is even, a theorem due to Micallef and Wang [30] 
states that if $\widetilde{M}$ has positive isotropic curvature, then $H^{2}(\widetilde{M}, \mathbb{R})=0$. Hence $\widetilde{M}$ cannot be a Kähler manifold. This together with Theorem B implies that $M$ is diffeomorphic to a spherical space form. q.e.d.

\section{Manifolds of positive scalar curvature}

In this section, we will give the proof of Theorem 1. More generally, we will prove Theorem 8 . We first prove the following lemma for compact manifolds.

Lemma 5. Let $M^{n}$ be an $n(\geq 4)$-dimensional compact Riemannian manifold. Denote by $R^{(k)}(\cdot)$ and $R^{(k, s)}(\cdot)$ the $k$-th scalar curvature and $(k, s)$-curvature of $M$. If one of the following conditions holds:

(i) $R_{\min }^{(k)}>\left(k^{2}+k-\frac{24}{7}\right) K_{\max }$ for some integer $k \in[3, n-1]$,

(ii) $R_{\min }^{(k, s)}>\frac{s\left(7 k^{2}+7 k-24\right)}{7(k+1)} K_{\max }$ for some integers $k \in[3, n-1]$ and $s \in[2, k+1]$,

then $\pi_{k}(M)=0$ for $2 \leq k \leq\left[\frac{n}{2}\right]$. In particular, if $M$ is simply connected, then $M$ is homeomorphic to a sphere.

Proof. (i) It follows from (2.7) that

$$
R_{\min }^{(k)} \leq 2 K_{\min }+[k(k+1)-2] K_{\max } .
$$

Then we have

$$
K_{\min } \geq \frac{1}{2}\left[R_{\min }^{(k)}-\left(k^{2}+k-2\right) K_{\max }\right] .
$$

Suppose $\left\{e_{1}, e_{2}, e_{3}, e_{4}\right\}$ is an orthonormal four-frame. From (2.2), (3.1), and the assumption we get

$$
\begin{aligned}
& R_{1313}+R_{1414}+R_{2323}+R_{2424}-2 R_{1234} \\
\geq & \frac{1}{2}\left\{R_{\min }^{(k)}-[k(k+1)-8] K_{\max }\right\}-\frac{4}{3}\left(K_{\max }-K_{\min }\right) \\
\geq & \frac{1}{2}\left\{R_{\min }^{(k)}-[k(k+1)-8] K_{\max }\right\}-\frac{2}{3}\left[k(k+1) K_{\max }-R_{\min }^{(k)}\right] \\
\geq & \frac{7}{6}\left[R_{\min }^{(k)}-\left(k^{2}+k-\frac{24}{7}\right) K_{\max }\right]
\end{aligned}
$$

Hence $M$ has positive isotropic curvature. By a result due to Micallef and Moore [29], we have $\pi_{k}(M)=0$ for $2 \leq k \leq\left[\frac{n}{2}\right]$. In particular, if $M$ is simply connected, then $M$ is homeomorphic to a sphere.

(ii) By Definition 1, we have

$$
\frac{R_{\min }^{(k)}}{k(k+1)} \geq \frac{R_{\min }^{(k, s)}}{k s} .
$$


This together with the assumption implies

$$
R_{\min }^{(k)} \geq \frac{k+1}{s} R_{\min }^{(k, s)}>\left(k^{2}+k-\frac{24}{7}\right) K_{\max } .
$$

Then the assertion follows from (i).

This completes the proof of Lemma 5 .

q.e.d.

By taking $k=n-1$ in Lemma 5, we have the following.

Theorem 6. Let $M^{n}$ be an $n(\geq 4)$-dimensional compact Riemannian manifold. Denote by $\operatorname{Ric}^{[s]}(\cdot)$ and $R_{0}(\cdot)$ the $s$-th weak Ricci curvature and normalized scalar curvature of $M$. If one of the following conditions holds:

(i) $R i c_{\min }^{[s]}>\frac{s\left(7 n^{2}-7 n-24\right)}{7 n} K_{\max }$ for some integer $s \in[2, n]$,

(ii) $R_{0}>\left[1-\frac{24}{7 n(n-1)}\right] K_{\max }$,

then $\pi_{k}(M)=0$ for $2 \leq k \leq\left[\frac{n}{2}\right]$. In particular, if $M$ is simply connected, then $M$ is homeomorphic to a sphere.

Corollary 1. Let $M^{n}$ be an $n$-dimensional compact and simply connected Riemannian manifold, where $4 \leq n \leq 6$. Denote by $R_{0}$ the normalized scalar curvature of $M$. If $R_{0}>\left[1-\frac{24}{7 n(n-1)}\right] K_{\max }$, then $M$ is diffeomorphic to $S^{n}$.

Proof. It follows from Theorem 6 that $M$ has positive isotropic curvature. A theorem due to Hamilton [24] says that a 4-dimensional compact simply connected manifold with positive isotropic curvature is diffeomorphic to $S^{4}$. It is well known that there is only one differentiable structure on $S^{n}, n=5,6$. This together with Theorem 6 implies $M$ is diffeomorphic to $S^{n}$ for $n=5,6$. This proves the corollary. q.e.d.

Lemma 6. Let $M^{n}$ be an $n(\geq 4)$-dimensional compact Riemannian manifold. Denote by $R^{(k)}(\cdot)$ and $R^{(k, s)}(\cdot)$ the $k$-th scalar curvature and $(k, s)$-curvature of $M$, respectively. If one of the following conditions holds:

(i) $R_{\min }^{(k)}>\left(k^{2}+k-\frac{12}{5}\right) K_{\max }$ for some integer $k \in[2, n-1]$,

(ii) $R_{\min }^{(k, s)}>\frac{s\left(5 k^{2}+5 k-12\right)}{5(k+1)} K_{\max }$ for some integers $k \in[2, n-1]$ and $s \in[2, k+1]$,

then $M$ is diffeomorphic to a spherical space form. 
Proof. (i) Suppose $\left\{e_{1}, e_{2}, e_{3}, e_{4}\right\}$ is an orthonormal four-frame and $\lambda \in \mathbb{R}$. From (2.2) and (3.1) we obtain

$$
\begin{aligned}
& R_{1313}+R_{2323}-\left|R_{1234}\right| \\
\geq & \frac{1}{2}\left[R_{\min }^{(k)}-2 \sum_{i<j \neq 3}^{k+1} R_{i j i j}\right]-\frac{2}{3}\left(K_{\max }-K_{\min }\right) \\
\geq & \frac{1}{2}\left[R_{\min }^{(k)}-\left(k^{2}+k-4\right) K_{\max }\right]-\frac{1}{3}\left[k(k+1) K_{\max }-R_{\min }^{(k)}\right] \\
\geq & \frac{5}{6}\left[R_{\min }^{(k)}-\left(k^{2}+k-\frac{12}{5}\right) K_{\max }\right] .
\end{aligned}
$$

Using the same argument as above, we get

$$
R_{1414}+R_{2424}-\left|R_{1234}\right| \geq \frac{5}{6}\left[R_{\min }^{(k)}-\left(k^{2}+k-\frac{12}{5}\right) K_{\max }\right] .
$$

From (3.5), (3.6), and the assumption, we have

$$
\begin{aligned}
& R_{1313}+\lambda^{2} R_{1414}+R_{2323}+\lambda^{2} R_{2424}-2 \lambda R_{1234} \\
\geq & R_{1313}+R_{2323}-\left|R_{1234}\right|+\lambda^{2}\left(R_{1414}+R_{2424}-\left|R_{1234}\right|\right) \\
\geq & \frac{5\left(1+\lambda^{2}\right)}{6}\left[R_{\min }^{(k)}-\left(k^{2}+k-\frac{12}{5}\right) K_{\max }\right] \\
> & 0 .
\end{aligned}
$$

This together with Theorem A implies that $M$ is diffeomorphic to a spherical space form.

(ii) From (3.3) and the assumption we know that

$$
R_{\min }^{(k)} \geq \frac{k+1}{s} R_{\min }^{(k, s)}>\left(k^{2}+k-\frac{12}{5}\right) K_{\max } .
$$

Hence the conclusion follows from (i).

This proves Lemma 6 .

q.e.d.

Lemma 7. Let $M^{n}$ be an $n(\geq 4)$-dimensional compact Riemannian manifold. If its $k$-th Ricci curvature satisfies one of the following conditions:

(i) $R i c_{\min }^{(k)}>\frac{5 k-6}{5 k-1} \operatorname{Ric}_{\max }^{(k+1)}$;

(ii) $R i c_{\min }^{(k)}>\frac{(5 k-6)(k+1)}{(5 k-1) k} \operatorname{Ric}_{\max }^{(k)}$,

where $k$ is some integer in $[2, n-2]$, then $M$ is diffeomorphic to a spherical space form.

Proof. (i) From (2.4), we obtain

$$
K_{\max } \leq R i c_{\max }^{(k+1)}-R i c_{\min }^{(k)},
$$

and

$$
R i c_{\min }^{(k)} \leq K_{\min }+(k-1) K_{\max }
$$


which implies

$$
\begin{aligned}
K_{\min } & \geq R i c_{\min }^{(k)}-(k-1) K_{\max } \\
& \geq k \operatorname{Ric} c_{\min }^{(k)}-(k-1) \operatorname{Ric}_{\max }^{(k+1)} .
\end{aligned}
$$

Suppose $\left\{e_{1}, e_{2}, e_{3}, e_{4}\right\}$ is an orthonormal four-frame and $\lambda \in \mathbb{R}$. Then we have from $(2.2),(2.4),(3.9)$, and (3.10) that

$$
\begin{aligned}
& R_{1313}+R_{2323}-\left|R_{1234}\right| \\
\geq & R i c_{\min }^{(k)}-\sum_{i=3}^{k+1} R_{i 3 i 3}-\frac{2}{3}\left(K_{\max }-K_{\min }\right) \\
\geq & R i c_{\min }^{(k)}-\left(k-\frac{4}{3}\right)\left[R i c_{\max }^{(k+1)}-R i c_{\min }^{(k)}\right] \\
& +\frac{2}{3}\left[k R i c_{\min }^{(k)}-(k-1) R i c_{\max }^{(k+1)}\right] \\
\geq & \frac{5 k-1}{3}\left[R i c_{\min }^{(k)}-\frac{5 k-6}{5 k-1} R i c_{\max }^{(k+1)}\right] .
\end{aligned}
$$

Similarly, we get

$$
R_{1414}+R_{2424}-\left|R_{1234}\right| \geq \frac{5 k-1}{3}\left[R i c_{\min }^{(k)}-\frac{5 k-6}{5 k-1} R i c_{\max }^{(k+1)}\right] .
$$

From (3.11), (3.12), and the assumption we obtain

$$
\begin{aligned}
& R_{1313}+\lambda^{2} R_{1414}+R_{2323}+\lambda^{2} R_{2424}-2 \lambda R_{1234} \\
\geq & R_{1313}+R_{2323}-\left|R_{1234}\right|+\lambda^{2}\left(R_{1414}+R_{2424}-\left|R_{1234}\right|\right) \\
\geq & \frac{\left(1+\lambda^{2}\right)(5 k-1)}{3}\left[R i c_{\min }^{(k)}-\frac{5 k-6}{5 k-1} R i c_{\max }^{(k+1)}\right]
\end{aligned}
$$

This together with Theorem A implies that $M$ is diffeomorphic to a spherical space form.

(ii) From (2.4) we have

$$
\frac{R i c_{\max }^{(k)}}{k} \geq \frac{R i c_{\max }^{(k+1)}}{k+1}
$$

which together with the assumption implies

$$
0<R i c_{\min }^{(k)}-\frac{(5 k-6)(k+1)}{(5 k-1) k} R i c_{\max }^{(k)} \leq R i c_{\min }^{(k)}-\frac{5 k-6}{5 k-1} R i c_{\max }^{(k+1)} .
$$

Hence the assertion follows from (i).

This proves Lemma 7.

q.e.d.

Taking $k=n-2$ in condition (i) of Lemma 7, we have the following theorem. 
Theorem 7. Let $M^{n}$ be an $n(\geq 4)$-dimensional compact Riemannian manifold. If its $(n-2)$-th Ricci curvature and Ricci curvature satisfy

$$
R i c_{\min }^{(n-2)}>\frac{5 n-16}{5 n-11} R i c_{\max },
$$

then $M$ is diffeomorphic to a spherical space form. In particular, if $M$ is simply connected, then $M$ is diffeomorphic to $S^{n}$.

Lemma 8. Let $M^{n}$ be an $n(\geq 4)$-dimensional compact Riemannian manifold. Denote by $\operatorname{Ric}^{(k)}(\cdot), R^{(k)}(\cdot)$, and $R^{(k, s)}(\cdot)$ the $k$-th Ricci curvature, $k$-th scalar curvature, and $(k, s)$-curvature of $M$, respectively. If one of the following conditions holds:

(i) $\operatorname{Ric}_{\min }^{(k)}>\frac{5 k-6}{5 k^{2}+9 k-8} R_{\max }^{(k+1)}$ for some integer $k \in[2, n-2]$,

(ii) $R i c_{\min }^{(k)}>\frac{(k+2)(5 k-6)}{s\left(5 k^{2}+9 k-8\right)} R_{\max }^{(k+1, s)}$ for some integers $k \in[2, n-2]$ and $s \in[2, k+2]$,

then $M$ is diffeomorphic to a spherical space form.

Proof. (i) It follows from (2.4) and (2.7) that

$$
K_{\max } \leq \frac{1}{2}\left[R_{\max }^{(k+1)}-(k+3) R i c_{\min }^{(k)}\right],
$$

and

$$
\operatorname{Ric}_{\min }^{(k)} \leq K_{\min }+(k-1) K_{\max }
$$

Then we have

$$
\begin{aligned}
K_{\min } & \geq \operatorname{Ric}_{\min }^{(k)}-(k-1) K_{\max } \\
& \geq \frac{1}{2}\left[\left(k^{2}+2 k-1\right) \operatorname{Ric}_{\min }^{(k)}-(k-1) R_{\max }^{(k+1)}\right] .
\end{aligned}
$$

Suppose $\left\{e_{1}, e_{2}, e_{3}, e_{4}\right\}$ is an orthonormal four-frame and $\lambda \in \mathbb{R}$. Combining (2.2), (2.4), (3.15), and (3.16), we have

$$
\begin{aligned}
& R_{1313}+R_{2323}-\left|R_{1234}\right| \\
\geq & R i c_{\min }^{(k)}-\sum_{i=3}^{k+1} R_{i 3 i 3}-\frac{2}{3}\left(K_{\max }-K_{\min }\right) \\
\geq & R i c_{\min }^{(k)}-\left(\frac{k}{2}-\frac{2}{3}\right)\left[R_{\max }^{(k+1)}-(k+3) R i c_{\min }^{(k)}\right] \\
& +\frac{1}{3}\left[\left(k^{2}+2 k-1\right) R i c_{\min }^{(k)}-(k-1) R_{\max }^{(k+1)}\right] \\
\geq & \frac{5 k^{2}+9 k-8}{6}\left[R i c_{\min }^{(k)}-\frac{5 k-6}{5 k^{2}+9 k-8} R_{\max }^{(k+1)}\right] .
\end{aligned}
$$

By a similar argument, we obtain

$$
R_{1414}+R_{2424}-\left|R_{1234}\right| \geq \frac{5 k^{2}+9 k-8}{6}\left[R i c_{\min }^{(k)}-\frac{5 k-6}{5 k^{2}+9 k-8} R_{\max }^{(k)}\right] .
$$


From (3.17), (3.18), and the assumption we obtain

$$
\begin{aligned}
& R_{1313}+\lambda^{2} R_{1414}+R_{2323}+\lambda^{2} R_{2424}-2 \lambda R_{1234} \\
\geq & R_{1313}+R_{2323}-\left|R_{1234}\right|+\lambda^{2}\left(R_{1414}+R_{2424}-\left|R_{1234}\right|\right) \\
\geq & \frac{\left(1+\lambda^{2}\right)\left(5 k^{2}+9 k-8\right)}{6}\left[R i c_{\min }^{(k)}-\frac{5 k-6}{5 k^{2}+9 k-8} R_{\max }^{(k)}\right] \\
> & 0 .
\end{aligned}
$$

This together with Theorem A implies that $M$ is diffeomorphic to a spherical space form.

(ii) We get from (2.5) and (2.7) that

$$
\frac{R_{\max }^{(k+1, s)}}{s(k+1)} \geq \frac{R_{\max }^{(k+1)}}{(k+1)(k+2)},
$$

which together with the assumption implies

$$
\begin{aligned}
R i c_{\min }^{(k)} & >\frac{(k+2)(5 k-6)}{s\left(5 k^{2}+9 k-8\right)} R_{\max }^{(k+1, s)} \\
& \geq \frac{5 k-6}{5 k^{2}+9 k-8} R_{\max }^{(k+1)} .
\end{aligned}
$$

The assertion follows from (i).

This proves the lemma.

q.e.d.

Theorem 8. Let $M^{n}$ be an $n(\geq 4)$-dimensional compact Riemannian manifold. Denote by $\operatorname{Ric}^{[s]}(\cdot), \operatorname{Ric}^{(k)}(\cdot)$, and $K(\cdot)$ the $s$-th weak Ricci curvature, $k$-th Ricci curvature, and sectional curvature of $M$, respectively. Suppose one of the following conditions holds:

(i) $R i c_{\min }^{[s]}>\frac{s\left(5 n^{2}-5 n-12\right)}{5 n} K_{\max }$ for some integer $s \in[2, n]$;

(ii) $R i c_{\min }^{[s]}>\frac{s\left(n^{2}+2 n+3\right)}{n(n+1)} R i c_{\max }^{(n-2)}$ for some integer $s \in[2, n]$;

(iii) $K_{\min }>\frac{1}{s(n-1)+6} R i c_{\max }^{[s]}$ for some integer $s \in[2, n]$;

(iv) $R i c_{\min }^{(n-2)}>\frac{n(5 n-16)}{s\left(5 n^{2}-11 n-6\right)}$ Ric $c_{\max }^{[s]}$ for some integer $s \in[2, n]$.

Then the normalized Ricci flow with initial metric $g_{0}$,

$$
\frac{\partial}{\partial t} g(t)=-2 R i c_{g(t)}+\frac{2}{n} r_{g(t)} g(t)
$$

exists for all time and converges to a constant curvature metric as $t \rightarrow \infty$. Moreover, $M$ is diffeomorphic to a spherical space form. In particular, if $M$ is simply connected, then $M$ is diffeomorphic to $S^{n}$.

Proof. (i) Taking $k=n-1$ in condition (ii) of Lemma 6, we get the conclusion. 
(ii) Since

$$
\begin{aligned}
K_{\min } & \geq \frac{1}{2}\left[R-(n+1) R i c_{\max }^{(n-2)}\right] \\
& \geq \frac{1}{2}\left[\frac{n R i c_{\min }^{[s]}}{s}-(n+1) R i c_{\max }^{(n-2)}\right]
\end{aligned}
$$

we obtain

$$
\begin{aligned}
K_{\max } & \leq R i c_{\max }^{(n-2)}-(n-3) K_{\min } \\
& \leq \frac{n^{2}-2 n-1}{2} \operatorname{Ric}_{\max }^{(n-2)}-\frac{n(n-3)}{2 s} \operatorname{Ric}_{\min }^{[s]}
\end{aligned}
$$

Suppose $\left\{e_{1}, e_{2}, e_{3}, e_{4}\right\}$ is an orthonormal four-frame and $\lambda \in \mathbb{R}$. It follows from (2.2), (3.22), and (3.23) that

$$
\begin{aligned}
& R_{1313}+R_{2323}-\left|R_{1234}\right| \\
\geq & 2 K_{\min }-\frac{2}{3}\left(K_{\max }-K_{\min }\right) \\
\geq & \frac{4}{3}\left[\frac{n R i c_{\min }^{[s]}}{s}-(n+1) R i c_{\max }^{(n-2)}\right] \\
& -\frac{2}{3}\left[\frac{n^{2}-2 n-1}{2} R i c_{\max }^{(n-2)}-\frac{n(n-3)}{2 s} R i c_{\min }^{[s]}\right] \\
\geq & \frac{1}{3}\left[\frac{n(n+1)}{s} R i c_{\min }^{[s]}-\left(n^{2}+2 n+3\right) R i c_{\max }^{(n-2)}\right] .
\end{aligned}
$$

Similarly, we get

$R_{1414}+R_{2424}-\left|R_{1234}\right| \geq \frac{1}{3}\left[\frac{n(n+1)}{s} \operatorname{Ric}_{\min }^{[s]}-\left(n^{2}+2 n+3\right) R i c_{\max }^{(n-2)}\right]$.

From (3.24), (3.25), and the assumption we obtain

$$
\begin{aligned}
& R_{1313}+\lambda^{2} R_{1414}+R_{2323}+\lambda^{2} R_{2424}-2 \lambda R_{1234} \\
\geq & R_{1313}+R_{2323}-\left|R_{1234}\right|+\lambda^{2}\left(R_{1414}+R_{2424}-\left|R_{1234}\right|\right) \\
\geq & \frac{1+\lambda^{2}}{3}\left[\frac{n(n+1)}{s} \operatorname{Ric}_{\min }^{[s]}-\left(n^{2}+2 n+3\right) \operatorname{Ric}_{\max }^{(n-2)}\right] \\
> & 0 .
\end{aligned}
$$

This together with Theorem A implies $M$ is diffeomorphic to a spherical space form.

(iii) By Definition 1, we get

$$
K_{\max } \leq \frac{1}{2}\left\{R i c_{\max }^{[s]}-[s(n-1)-2] K_{\min }\right\}
$$


Suppose $\left\{e_{1}, e_{2}, e_{3}, e_{4}\right\}$ is an orthonormal four-frame and $\lambda \in \mathbb{R}$. It follows from (2.2) and (3.27) that

$$
\begin{aligned}
& R_{1313}+R_{2323}-\left|R_{1234}\right| \\
\geq & 2 K_{\min }-\frac{2}{3}\left(K_{\max }-K_{\min }\right) \\
\geq & \frac{8}{3} K_{\min }-\frac{1}{3}\left[R i c_{\max }^{[s]}-(s n-s-2) K_{\min }\right] \\
\geq & \frac{1}{3}\left[(s n-s+6) K_{\min }-R i c_{\max }^{[s]}\right] .
\end{aligned}
$$

A similar discussion implies that

$$
R_{1414}+R_{2424}-\left|R_{1234}\right| \geq \frac{1}{3}\left[(s n-s+6) K_{\min }-R i c_{\max }^{[s]}\right] .
$$

From (3.28), (3.29), and the assumption we obtain

$$
\begin{aligned}
& R_{1313}+\lambda^{2} R_{1414}+R_{2323}+\lambda^{2} R_{2424}-2 \lambda R_{1234} \\
\geq & R_{1313}+R_{2323}-\left|R_{1234}\right|+\lambda^{2}\left(R_{1414}+R_{2424}-\left|R_{1234}\right|\right) \\
\geq & \frac{1+\lambda^{2}}{3}\left[(\text { sn }-s+6) K_{\min }-R i c_{\max }^{[s]}\right] \\
> & 0 .
\end{aligned}
$$

This together with Theorem A implies that $M$ is diffeomorphic to a spherical space form.

(iv) The assertion follows by taking $k=n-2$ in (ii) of Lemma 8 .

This proves the theorem.

We are now in a position to prove Theorem 1 .

Proof of Theorem 1. (i) If $n=3$, for any unit tangent vector $u \in U_{x} M$ at $x \in M$, we choose an orthonormal three-frame $\left\{e_{1}, e_{2}, e_{3}\right\}$ such that $e_{3}=u$. Then from the assumption we obtain

$$
\begin{aligned}
\operatorname{Ric}(u) & =R_{1313}+R_{2323} \\
& =\frac{1}{2}\left(R-2 R_{1212}\right) \\
& \geq \frac{1}{2}\left(R-2 K_{\max }\right) \\
& >0 .
\end{aligned}
$$

This together with Hamilton's theorem [23] implies that $M$ is diffeomorphic to a spherical space form. When $n \geq 4$, the assertion follows by taking $k=n-1$ in (i) of Lemma 6 .

(ii) If $n=3$, the assertion follows from Hamilton's work [23]. Thus from now on we assume that $n \geq 4$. By taking $s=n$ in (iii) of Theorem 8 , we conclude that $M$ is diffeomorphic to a spherical space form.

This proves Theorem 1.

q.e.d. 
In 1990, Yau [54] proposed the following conjecture (see also $[\mathbf{3 9}, \mathbf{5 5}]$ ).

Yau Conjecture 1. Let $M^{n}$ be a compact and simply connected Riemannian manifold. Denote by $R_{0}$ the normalized scalar curvature of $M$. If $K_{\min }>\frac{n-1}{n+2} R_{0}$, then $M$ is diffeomorphic to $S^{n}$.

If $n=2,3$, the answer is affirmative. If the pinching constant in Yau Conjecture 1 is replaced by $\eta_{n}=\frac{n^{2}-n}{n^{2}-n+6}$, Theorem 1 gives an affirmative answer. The following example shows that $\frac{n-1}{n+2}$ is the best possible pinching constant for the conjecture in even dimensions $(n \geq 4)$.

Example 1. Let $R_{0}$ be the normalized scalar curvature of a Riemannian manifold. By a direct computation, we have the normalized scalar curvatures of the compact rank one symmetric spaces (CROSS) with standard metrics:

$$
\begin{aligned}
& R_{0}\left(\mathbb{C} P^{m}\right)=\frac{m+1}{4 m-2}, \operatorname{dim}_{\mathbb{R}}\left(\mathbb{C} P^{m}\right)=2 m, m \geq 2 ; \\
& R_{0}\left(\mathbb{H} P^{m}\right)=\frac{m+2}{4 m-1}, \operatorname{dim}_{\mathbb{R}}\left(\mathbb{H} P^{m}\right)=4 m, m \geq 2 ; \\
& R_{0}\left(\mathbb{O} P^{2}\right)=\frac{3}{5}, \operatorname{dim}_{\mathbb{R}}\left(\mathbb{O} P^{2}\right)=16 .
\end{aligned}
$$

On the other hand,

$$
K_{\min }\left(\mathbb{C} P^{m}\right)=K_{\min }\left(\mathbb{H} P^{m}\right)=K_{\min }\left(\mathbb{O} P^{2}\right)=\frac{1}{4},
$$

and these are not homeomorphic to $S^{n}$. Therefore, $\frac{n-1}{n+2}$ is the best possible pinching constant for Yau Conjecture 1 in even dimensions $(\geq 4)$.

Yau Conjecture 2. Let $M^{n}$ be a compact and simply connected Riemannian manifold. Denote by $R_{0}$ the normalized scalar curvature of $M$. If $K_{M} \geq \frac{n-1}{n+2}$ and $R_{0} \leq 1$, then $M$ is either diffeomorphic to $S^{n}$, or isometric to the complex projective space $\mathbb{C} P^{m}$ with $n=2 m$.

Recently the authors [52] proved the following optimal rigidity theorem for Einstein manifolds, which provides evidence for Yau Conjectures 1 and 2 .

Theorem 9. Let $M$ be an $n(\geq 4)$-dimensional compact Einstein manifold with normalized scalar curvature $R_{0}:=c$. If $K_{\min } \geq \frac{n-1}{n+2} R_{0}>$ 0 , then $M$ is locally symmetric. In particular, if $M$ is simply connected, then $M$ is isometric to either the standard $n$-sphere $S^{n}\left(\frac{1}{\sqrt{c}}\right)$ or the complex projective space $\mathbb{C} P^{m}(c)$.

Motivated by Theorem 1 and Example 1, we would like to propose the following conjectures.

Conjecture 1. Let $M^{n}(n \geq 4)$ be a compact Riemannian manifold. If $R_{0}>\frac{3}{5} K_{\max }$, then $M$ is diffeomorphic to a spherical space form. In particular, if $M$ is simply connected, then $M$ is diffeomorphic to $S^{n}$. 
Conjecture 2. Let $M^{n}(n \geq 4)$ be an even dimensional compact and simply connected Riemannian manifold. If $K_{M} \leq 1$ and $R_{0} \geq c_{n}$, where

$$
c_{n}= \begin{cases}\frac{n+2}{4(n-1)} & \text { for } n=4 \text { or } 4 k+2, \quad k \in \mathbb{Z}^{+}, \\ \frac{n+8}{4(n-1)} & \text { for } n=4 k, \quad k \in \mathbb{Z}^{+} \bigcap[2, \infty) \text { and } k \neq 4, \\ \frac{3}{5} & \text { for } n=16,\end{cases}
$$

then $M$ is either diffeomorphic to $S^{n}$, or isometric to a compact rank one symmetric space.

Proof of Theorem 2. (i) If $n=3$, the assertion follows from (i) of Theorem 1. If $n \geq 4$, the conclusion follows from (ii) of Theorem 8 by taking $s=n$.

(ii) If $n=3$, it follows from Hamilton's work [23]. If $n \geq 4$, by taking $k=n-2$ in (i) of Lemma 8, we get the conclusion.

This completes the proof of Theorem 2 .

q.e.d.

\section{Sphere theorems for compact submanifolds}

In this section, we extend the sphere theorems in Section 3 to submanifolds in Riemannian manifolds with arbitrary codimension. For compact submanifolds, we prove the following lemma.

Lemma 9. Let $M^{n}$ be an $n(\geq 4)$-dimensional compact submanifold in an $N$-dimensional Riemannian manifold $\bar{M}^{N}$. Assume that $M$ satisfies one of the following conditions:

(i) $S<\frac{7}{6}\left[\bar{R}_{\min }^{(k)}-\left(k^{2}+k-\frac{24}{7}\right) \bar{K}_{\max }\right]+\frac{n^{2} H^{2}}{n-2}$ for some integer $k \in$ $[3, N-1]$

(ii) $S<\frac{7(k+1)}{6 s}\left[\bar{R}_{\min }^{(k, s)}-\frac{s\left(7 k^{2}+7 k-24\right)}{7(k+1)} \bar{K}_{\max }\right]+\frac{n^{2} H^{2}}{n-2}$ for some integers $k \in[3, N-1]$ and $s \in[2, k+1]$.

Then $\pi_{k}(M)=0$ for $2 \leq k \leq\left[\frac{n}{2}\right]$. In particular, if $M$ is simply connected, then $M$ is homeomorphic to a sphere.

Proof. (i) Since

$$
\bar{R}_{\min }^{(k)} \leq 2 \bar{K}_{\min }+[k(k+1)-2] \bar{K}_{\max },
$$

we have

$$
\bar{K}_{\min } \geq \frac{1}{2}\left[\bar{R}_{\min }^{(k)}-\left(k^{2}+k-2\right) \bar{K}_{\max }\right] .
$$

Setting $S_{\alpha}=\sum_{i, j=1}^{n}\left(h_{i j}^{\alpha}\right)^{2}$, we know that

$$
\left(\sum_{i=1}^{n} h_{i i}^{\alpha}\right)^{2}=(n-2)\left[\sum_{i=1}^{n}\left(h_{i i}^{\alpha}\right)^{2}+\sum_{i \neq j}\left(h_{i j}^{\alpha}\right)^{2}+\frac{\left(\sum_{i=1}^{n} h_{i i}^{\alpha}\right)^{2}}{n-2}-S_{\alpha}\right] .
$$


Note that for all distinct $p, q, m, l$

$$
\begin{aligned}
\left(\sum_{i=1}^{n} h_{i i}^{\alpha}\right)^{2} & \leq(n-2)\left[\left(h_{p p}^{\alpha}+h_{q q}^{\alpha}\right)^{2}+\left(h_{m m}^{\alpha}+h_{l l}^{\alpha}\right)^{2}+\sum_{i \neq p, q, m, l}\left(h_{i i}^{\alpha}\right)^{2}\right] \\
& =(n-2)\left[\sum_{i=1}^{n}\left(h_{i i}^{\alpha}\right)^{2}+2 h_{p p}^{\alpha} h_{q q}^{\alpha}+2 h_{m m}^{\alpha} h_{l l}^{\alpha}\right] .
\end{aligned}
$$

This together with (4.2) implies

$$
2 h_{p p}^{\alpha} h_{q q}^{\alpha}+2 h_{m m}^{\alpha} h_{l l}^{\alpha} \geq \sum_{i \neq j}\left(h_{i j}^{\alpha}\right)^{2}+\frac{\left(\sum_{i=1}^{n} h_{i i}^{\alpha}\right)^{2}}{n-2}-S_{\alpha}
$$

for all distinct $p, q, m, l$. Suppose $\left\{e_{1}, e_{2}, e_{3}, e_{4}\right\}$ is an orthonormal fourframe and $\lambda \in \mathbb{R}$. From (2.1), (2.3), (4.1), (4.3), and the assumption we get

$$
\begin{aligned}
& R_{1313}+R_{1414}+R_{2323}+R_{2424}-2 R_{1234} \\
= & \bar{R}_{1313}+\bar{R}_{1414}+\bar{R}_{2323}+\bar{R}_{2424}-2 \bar{R}_{1234} \\
& +\sum_{\alpha}\left[h_{11}^{\alpha} h_{33}^{\alpha}+h_{22}^{\alpha} h_{44}^{\alpha}+h_{22}^{\alpha} h_{33}^{\alpha}+h_{11}^{\alpha} h_{44}^{\alpha}\right. \\
& \left.-\left(h_{13}^{\alpha}\right)^{2}-\left(h_{23}^{\alpha}\right)^{2}-\left(h_{24}^{\alpha}\right)^{2}-\left(h_{14}^{\alpha}\right)^{2}-2\left(h_{13}^{\alpha} h_{24}^{\alpha}-h_{14}^{\alpha} h_{23}^{\alpha}\right)\right] \\
\geq & \frac{1}{2}\left\{\bar{R}_{\min }^{(k)}-[k(k+1)-8] \bar{K}_{\max }\right\}-\frac{4}{3}\left(\bar{K}_{\max }-\bar{K}_{\min }\right) \\
& +\sum_{\alpha}\left[\sum_{i \neq j}\left(h_{i j}^{\alpha}\right)^{2}+\frac{\left(\sum_{i=1}^{n} h_{i i}^{\alpha}\right)^{2}}{n-2}-S_{\alpha}\right. \\
& \left.-2\left(h_{13}^{\alpha}\right)^{2}-2\left(h_{23}^{\alpha}\right)^{2}-2\left(h_{24}^{\alpha}\right)^{2}-2\left(h_{14}^{\alpha}\right)^{2}\right] \\
\geq & \frac{1}{2}\left[\bar{R}_{\min }^{(k)}-\left(k^{2}+k-8\right) \bar{K}_{\max }\right] \\
& -\frac{2}{3}\left[k(k+1) \bar{K}_{\max }-\bar{R}_{\min }^{(k)}\right]+\frac{n^{2} H^{2}}{n-2}-S \\
\geq & \frac{7}{6}\left[\bar{R}_{\min }^{(k)}-\left(k^{2}+k-\frac{24}{7}\right) \bar{K}_{\max }\right]+\frac{n^{2} H^{2}}{n-2}-S \\
> & 0 .
\end{aligned}
$$

Therefore $M$ has positive isotropic curvature. From Micallef and Moore's theorem [29], we get $\pi_{k}(M)=0$ for $2 \leq k \leq\left[\frac{n}{2}\right]$. In particular, if $M$ is simply connected, then $M$ is homeomorphic to a sphere.

(ii) Notice that

$$
\frac{\bar{R}_{\min }^{(k)}}{k(k+1)} \geq \frac{\bar{R}_{\min }^{(k, s)}}{k s}
$$


We have

$$
\bar{R}_{\min }^{(k)}-\left(k^{2}+k-\frac{24}{7}\right) \bar{K}_{\max } \geq \frac{k+1}{s} \bar{R}_{\min }^{(k, s)}-\left(k^{2}+k-\frac{24}{7}\right) \bar{K}_{\max } .
$$

The assertion follows from (i), (4.6), and the assumption.

This proves Lemma 9.

q.e.d.

By taking $k=N-1$ in Lemma 9, we get the following theorem.

Theorem 10. Let $M^{n}$ be an $n(\geq 4)$-dimensional compact submanifold in an N-dimensional Riemannian manifold $\bar{M}^{N}$. Assume that $M$ satisfies one of the following conditions:

(i) $S<\frac{7}{6}\left[\bar{R}-\left(N^{2}-N-\frac{24}{7}\right) \bar{K}_{\max }\right]+\frac{n^{2} H^{2}}{n-2}$;

(ii) $S<\frac{7 N}{6 s}\left[\overline{\operatorname{Ric}}_{\min }^{[s]}-\frac{s\left(7 N^{2}-7 N-24\right)}{7 N} \bar{K}_{\max }\right]+\frac{n^{2} H^{2}}{n-2}$ for some integer $s \in[2, N]$.

Then $\pi_{k}(M)=0$ for $2 \leq k \leq\left[\frac{n}{2}\right]$. In particular, if $M$ is simply connected, then $M$ is homeomorphic to a sphere.

Lemma 10. Let $M^{n}$ be an $n(\geq 4)$-dimensional compact submanifold in an N-dimensional Riemannian manifold $\bar{M}^{N}$. Suppose that $M$ satisfies one of the following conditions:

(i) $S<\frac{5}{6}\left[\bar{R}_{\min }^{(k)}-\left(k^{2}+k-\frac{12}{5}\right) \bar{K}_{\max }\right]+\frac{n^{2} H^{2}}{n-1}$, for some integer $k \in$ $[2, N-1]$

(ii) $S<\frac{5(k+1)}{6 s}\left[\bar{R}_{\min }^{(k, s)}-\frac{s\left(5 k^{2}+5 k-12\right)}{5(k+1)} \bar{K}_{\max }\right]+\frac{n^{2} H^{2}}{n-1}$, for some integers $k \in[2, N-1]$ and $s \in[2, k+1]$.

Then $M$ is diffeomorphic to a spherical space form.

Proof. (i) Setting $S_{\alpha}=\sum_{i, j=1}^{n}\left(h_{i j}^{\alpha}\right)^{2}$, we have

$$
\left(\sum_{i=1}^{n} h_{i i}^{\alpha}\right)^{2}=(n-1)\left[\sum_{i=1}^{n}\left(h_{i i}^{\alpha}\right)^{2}+\sum_{i \neq j}\left(h_{i j}^{\alpha}\right)^{2}+\frac{\left(\sum_{i=1}^{n} h_{i i}^{\alpha}\right)^{2}}{n-1}-S_{\alpha}\right]
$$

Note that for $m \neq l$

$$
\begin{aligned}
\left(\sum_{i=1}^{n} h_{i i}^{\alpha}\right)^{2} & \leq(n-1)\left[\left(h_{m m}^{\alpha}+h_{l l}^{\alpha}\right)^{2}+\sum_{i \neq m, l}\left(h_{i i}^{\alpha}\right)^{2}\right] \\
& =(n-1)\left[\sum_{i=1}^{n}\left(h_{i i}^{\alpha}\right)^{2}+2 h_{m m}^{\alpha} h_{l l}^{\alpha}\right] .
\end{aligned}
$$

This together with (4.7) implies

$$
2 h_{m m}^{\alpha} h_{l l}^{\alpha} \geq \sum_{i \neq j}\left(h_{i j}^{\alpha}\right)^{2}+\frac{\left(\sum_{i=1}^{n} h_{i i}^{\alpha}\right)^{2}}{n-1}-S_{\alpha}
$$


for all distinct $m, l$, and the equality holds if and only if $h_{i i}^{\alpha}=h_{m m}^{\alpha}+h_{l l}^{\alpha}$ for all $i \neq m, l$. Suppose $\left\{e_{1}, e_{2}, e_{3}, e_{4}\right\}$ is an orthonormal four-frame and $\lambda \in \mathbb{R}$. From (2.1), (2.3), (4.1), and (4.8), we obtain

$$
\begin{aligned}
& R_{1313}+R_{2323}-\left|R_{1234}\right| \\
\geq & \frac{1}{2}\left[\bar{R}_{\min }^{(k)}-2 \sum_{A<B \neq 3}^{k+1} \bar{R}_{A B A B}\right]-\frac{2}{3}\left(\bar{K}_{\max }-\bar{K}_{\min }\right)+\sum_{\alpha}\left[h_{11}^{\alpha} h_{33}^{\alpha}\right. \\
& \left.+h_{22}^{\alpha} h_{33}^{\alpha}-\frac{3}{2}\left(h_{13}^{\alpha}\right)^{2}-\frac{3}{2}\left(h_{23}^{\alpha}\right)^{2}-\frac{1}{2}\left(h_{24}^{\alpha}\right)^{2}-\frac{1}{2}\left(h_{14}^{\alpha}\right)^{2}\right] \\
\geq & \frac{1}{2}\left[\bar{R}_{\min }^{(k)}-\left(k^{2}+k-4\right) \bar{K}_{\max }\right]-\frac{1}{3}\left[k(k+1) \bar{K}_{\max }-\bar{R}_{\min }^{(k)}\right] \\
& +\sum_{\alpha}\left[\sum_{i \neq j}\left(h_{i j}^{\alpha}\right)^{2}+\frac{\left(\sum_{i=1}^{n} h_{i i}^{\alpha}\right)^{2}}{n-1}-S_{\alpha}\right. \\
& \left.-\frac{3}{2}\left(h_{13}^{\alpha}\right)^{2}-\frac{3}{2}\left(h_{23}^{\alpha}\right)^{2}-\frac{1}{2}\left(h_{24}^{\alpha}\right)^{2}-\frac{1}{2}\left(h_{14}^{\alpha}\right)^{2}\right] \\
(4.9) \geq & \frac{5}{6}\left[\bar{R}_{\min }^{(k)}-\left(k^{2}+k-\frac{12}{5}\right) \bar{K}_{\max }\right]+\frac{n^{2} H^{2}}{n-1}-S .
\end{aligned}
$$

Similarly, we get

$$
\begin{aligned}
& R_{1414}+R_{2424}-\left|R_{1234}\right| \\
\geq & \frac{5}{6}\left[\bar{R}_{\min }^{(k)}-\left(k^{2}+k-\frac{12}{5}\right) \bar{K}_{\max }\right]+\frac{n^{2} H^{2}}{n-1}-S .
\end{aligned}
$$

From (4.9) and (4.10), we obtain

$$
\begin{aligned}
& R_{1313}+\lambda^{2} R_{1414}+R_{2323}+\lambda^{2} R_{2424}-2 \lambda R_{1234} \\
& \geq R_{1313}+R_{2323}-\left|R_{1234}\right|+\lambda^{2}\left(R_{1414}+R_{2424}-\left|R_{1234}\right|\right) \\
& (4.11) \geq\left(1+\lambda^{2}\right)\left\{\frac{5}{6}\left[\bar{R}_{\min }^{(k)}-\left(k^{2}+k-\frac{12}{5}\right) \bar{K}_{\max }\right]+\frac{n^{2} H^{2}}{n-1}-S\right\} \text {. }
\end{aligned}
$$

This together with Theorem A and the assumption implies $M$ is diffeomorphic to a spherical space form.

(ii) From (4.5) we have

$$
\bar{R}_{\min }^{(k)}-\left(k^{2}+k-\frac{12}{5}\right) \bar{K}_{\max } \geq \frac{k+1}{s} \bar{R}_{\min }^{(k, s)}-\left(k^{2}+k-\frac{12}{5}\right) \bar{K}_{\max } .
$$

Therefore the assertion follows from (i), (4.12), and the assumption.

This completes the proof.

q.e.d.

Lemma 11. Let $M^{n}$ be an $n(\geq 4)$-dimensional compact submanifold in an N-dimensional Riemannian manifold $\bar{M}^{N}$. Suppose that $M$ satisfies one of the following conditions:

(i) $S<\frac{5 k-1}{3}\left[\overline{\operatorname{Ric}}_{\min }^{(k)}-\frac{5 k-6}{5 k-1} \overline{\operatorname{Ric}}_{\max }^{(k+1)}\right]+\frac{n^{2} H^{2}}{n-1}$ for some integer $k \in$ $[2, N-2]$; 
(ii) $S<\frac{5 k-1}{3}\left[\overline{\operatorname{Ric}}_{\min }^{(k)}-\frac{(5 k-6)(k+1)}{(5 k-1) k} \overline{\operatorname{Ric}}_{\max }^{(k)}\right]+\frac{n^{2} H^{2}}{n-1}$, for some integer $k \in[2, N-2]$.

Then $M$ is diffeomorphic to a spherical space form.

Proof. Since

$$
\bar{K}_{\max } \leq \overline{R i c}_{\max }^{(k+1)}-\overline{\operatorname{Ric}}_{\min }^{(k)}
$$

and

$$
\overline{\operatorname{Ric}}_{\min }^{(k)} \leq \bar{K}_{\min }+(k-1) \bar{K}_{\max }
$$

we have

$$
\begin{aligned}
\bar{K}_{\min } & \geq \overline{\operatorname{Ric}}_{\min }^{(k)}-(k-1) \bar{K}_{\max } \\
& \geq k \overline{\operatorname{Ric}}_{\min }^{(k)}-(k-1) \overline{\operatorname{Ric}}_{\max }^{(k+1)}
\end{aligned}
$$

Suppose $\left\{e_{1}, e_{2}, e_{3}, e_{4}\right\}$ is an orthonormal four-frame and $\lambda \in \mathbb{R}$. From (2.1), (2.3), (4.8), (4.13), and (4.14) we get

$$
\begin{aligned}
& R_{1313}+R_{2323}-\left|R_{1234}\right| \\
\geq & \overline{R i c}_{\min }^{(k)}-\sum_{A=3}^{k+1} \bar{R}_{A 3 A 3}-\frac{2}{3}\left(\bar{K}_{\max }-\bar{K}_{\min }\right)+\sum_{\alpha}\left[h_{11}^{\alpha} h_{33}^{\alpha}\right. \\
& \left.+h_{22}^{\alpha} h_{33}^{\alpha}-\frac{3}{2}\left(h_{13}^{\alpha}\right)^{2}-\frac{3}{2}\left(h_{23}^{\alpha}\right)^{2}-\frac{1}{2}\left(h_{24}^{\alpha}\right)^{2}-\frac{1}{2}\left(h_{14}^{\alpha}\right)^{2}\right] \\
\geq & \overline{R i c}_{\min }^{(k)}-\left(k-\frac{4}{3}\right)\left[\overline{\operatorname{Ric}}_{\max }^{(k+1)}-\overline{R i c}_{\min }^{(k)}\right] \\
& +\frac{2}{3}\left[k \overline{R i c}_{\min }^{(k)}-(k-1) \overline{R i c}_{\max }^{(k+1)}\right] \\
& +\sum_{\alpha}\left[\sum_{i \neq j}\left(h_{i j}^{\alpha}\right)^{2}+\frac{\left(\sum_{i=1}^{n} h_{i i}^{\alpha}\right)^{2}}{n-1}-S_{\alpha}\right. \\
& \left.-\frac{3}{2}\left(h_{13}^{\alpha}\right)^{2}-\frac{3}{2}\left(h_{23}^{\alpha}\right)^{2}-\frac{1}{2}\left(h_{24}^{\alpha}\right)^{2}-\frac{1}{2}\left(h_{14}^{\alpha}\right)^{2}\right] \\
\geq & \frac{5 k-1}{3}\left[\overline{R i c}_{\min }^{(k)}-\frac{5 k-6}{5 k-1} \overline{R i c}_{\max }^{(k+1)}\right]+\frac{n^{2} H^{2}}{n-1}-S .
\end{aligned}
$$

Similarly, we have

$$
\begin{aligned}
& R_{1414}+R_{2424}-\left|R_{1234}\right| \\
\geq & \frac{5 k-1}{3}\left[\overline{R i c}_{\min }^{(k)}-\frac{5 k-6}{5 k-1} \overline{R i c}_{\max }^{(k+1)}\right]+\frac{n^{2} H^{2}}{n-1}-S .
\end{aligned}
$$


This together with (4.15) and the assumption implies

$$
\begin{aligned}
& R_{1313}+\lambda^{2} R_{1414}+R_{2323}+\lambda^{2} R_{2424}-2 \lambda R_{1234} \\
\geq & R_{1313}+R_{2323}-\left|R_{1234}\right|+\lambda^{2}\left(R_{1414}+R_{2424}-\left|R_{1234}\right|\right) \\
\geq & \left(1+\lambda^{2}\right)\left\{\frac{5 k-1}{3}\left[\overline{R i c}_{\min }^{(k)}-\frac{5 k-6}{5 k-1} \overline{R i c}_{\max }^{(k+1)}\right]+\frac{n^{2} H^{2}}{n-1}-S\right\} \\
(4.17)> & 0 .
\end{aligned}
$$

The assertion follows from Theorem A.

(ii) Since

$$
\frac{\overline{\operatorname{Ric}}_{\max }^{(k)}}{k} \geq \frac{\overline{\operatorname{Ric}}_{\max }^{(k+1)}}{k+1}
$$

we have

$$
\overline{\operatorname{Ric}}_{\min }^{(k)}-\frac{(5 k-6)(k+1)}{(5 k-1) k} \overline{\operatorname{Ric}}_{\max }^{(k)} \leq \overline{\operatorname{Ric}}_{\min }^{(k)}-\frac{5 k-6}{5 k-1} \overline{\operatorname{Ric}}_{\max }^{(k+1)} .
$$

The assertion follows from the assumption and (i).

This completes the proof.

q.e.d.

Taking $k=N-2$ in (i) of Lemma 11, we get the following theorem.

Theorem 11. Let $M^{n}$ be an $n(\geq 4)$-dimensional compact submanifold in an $N$-dimensional Riemannian manifold $\bar{M}^{N}$. If $S<\frac{5 N-11}{3}\left[\overline{\operatorname{Ric}}_{\mathrm{min}}^{(N-2)}\right.$ $\left.-\frac{5 N-16}{5 N-11} \overline{R i c}_{\max }\right]+\frac{n^{2} H^{2}}{n-1}$, then $M$ is diffeomorphic to a spherical space form. In particular, if $M$ is simply connected, then $M$ is diffeomorphic to $S^{n}$.

Lemma 12. Let $M^{n}$ be an $n(\geq 4)$-dimensional compact submanifold in an N-dimensional Riemannian manifold $\bar{M}^{N}$. Suppose that $M$ satisfies one of the following conditions:

(i) $S<\frac{5 k^{2}+9 k-8}{6}\left[\overline{R i c}_{\min }^{(k)}-\frac{5 k-6}{5 k^{2}+9 k-8} \bar{R}_{\max }^{(k+1)}\right]+\frac{n^{2} H^{2}}{n-1}$, for some integer $k \in[2, N-2]$;

(ii) $S<\frac{5 k^{2}+9 k-8}{6}\left[\overline{\operatorname{Ric}}_{\min }^{(k)}-\frac{(k+2)(5 k-6)}{s\left(5 k^{2}+9 k-8\right)} \bar{R}_{\max }^{(k+1, s)}\right]+\frac{n^{2} H^{2}}{n-1}$, for some integers $k \in[2, N-2]$ and $s \in[2, k+1]$.

Then $M$ is diffeomorphic to a spherical space form.

Proof. (i) It's seen from (2.8) and (2.9) that

$$
\bar{K}_{\max } \leq \frac{1}{2}\left[\bar{R}_{\max }^{(k+1)}-(k+3) \overline{\operatorname{Ric}}_{\min }^{(k)}\right],
$$

and

$$
\overline{\operatorname{Ric}}_{\min }^{(k)} \leq \bar{K}_{\min }+(k-1) \bar{K}_{\max },
$$


which implies

$$
\begin{aligned}
\bar{K}_{\min } & \geq \overline{\operatorname{Ric}}_{\min }^{(k)}-(k-1) \bar{K}_{\max } \\
& \geq \frac{1}{2}\left[\left(k^{2}+2 k-1\right) \overline{\operatorname{Ric}}_{\min }^{(k)}-(k-1) \bar{R}_{\max }^{(k+1)}\right] .
\end{aligned}
$$

Suppose $\left\{e_{1}, e_{2}, e_{3}, e_{4}\right\}$ is an orthonormal four-frame and $\lambda \in \mathbb{R}$. It follows from (2.1), (2.3), (4.8), (4.19), and (4.20) that

$$
\begin{aligned}
& R_{1313}+R_{2323}-\left|R_{1234}\right| \\
\geq & \overline{R i c}_{\min }^{(k)}-\sum_{A=3}^{k+1} \bar{R}_{A 3 A 3}-\frac{2}{3}\left(\bar{K}_{\max }-\bar{K}_{\min }\right)+\sum_{\alpha}\left[h_{11}^{\alpha} h_{33}^{\alpha}\right. \\
& \left.+h_{22}^{\alpha} h_{33}^{\alpha}-\frac{3}{2}\left(h_{13}^{\alpha}\right)^{2}-\frac{3}{2}\left(h_{23}^{\alpha}\right)^{2}-\frac{1}{2}\left(h_{24}^{\alpha}\right)^{2}-\frac{1}{2}\left(h_{14}^{\alpha}\right)^{2}\right] \\
\geq & \overline{R i c}_{\min }^{(k)}-\left(\frac{k}{2}-\frac{2}{3}\right)\left[\bar{R}_{\max }^{(k+1)}-(k+3) \overline{R i c}_{\min }^{(k)}\right] \\
& +\frac{1}{3}\left[\left(k^{2}+2 k-1\right) \overline{\operatorname{Ric}}_{\min }^{(k)}-(k-1) \bar{R}_{\max }^{(k+1)}\right]+\frac{n^{2} H^{2}}{n-1}-S \\
\geq & \frac{5 k^{2}+9 k-8}{6}\left[\overline{R i c}_{\min }^{(k)}-\frac{5 k-6}{5 k^{2}+9 k-8} \bar{R}_{\max }^{(k+1)}\right]+\frac{n^{2} H^{2}}{n-1}-S .
\end{aligned}
$$

By a similar computation, we get

$$
\begin{aligned}
& R_{1414}+R_{2424}-\left|R_{1234}\right| \\
\geq & \frac{5 k^{2}+9 k-8}{6}\left[\overline{R i c}_{\min }^{(k)}-\frac{5 k-6}{5 k^{2}+9 k-8} \bar{R}_{\max }^{(k+1)}\right]+\frac{n^{2} H^{2}}{n-1}-S .
\end{aligned}
$$

From (4.21) and (4.22), we obtain

$$
\begin{array}{ll} 
& R_{1313}+\lambda^{2} R_{1414}+R_{2323}+\lambda^{2} R_{2424}-2 \lambda R_{1234} \\
\geq & R_{1313}+R_{2323}-\left|R_{1234}\right|+\lambda^{2}\left(R_{1414}+R_{2424}-\left|R_{1234}\right|\right) \\
\geq & \left(1+\lambda^{2}\right)\left\{\frac{5 k^{2}+9 k-8}{6}\left[\overline{R i c}_{\min }^{(k)}-\frac{5 k-6}{5 k^{2}+9 k-8} \bar{R}_{\max }^{(k+1)}\right]\right. \\
& \left.+\frac{n^{2} H^{2}}{n-1}-S\right\} .
\end{array}
$$

From (4.23), Theorem A, and the assumption, we see that $M$ is diffeomorphic to a spherical space form.

(ii) It follows from (2.9) that

$$
\frac{\bar{R}_{\max }^{(k+1, s)}}{s(k+1)} \geq \frac{\bar{R}_{\max }^{(k+1)}}{(k+1)(k+2)},
$$

which implies

$$
\begin{gathered}
\overline{R i c}_{\min }^{(k)}-\frac{5 k-6}{5 k^{2}+9 k-8} \bar{R}_{\max }^{(k+1)} \\
\geq \overline{\operatorname{Ric}}_{\min }^{(k)}-\frac{(k+2)(5 k-6)}{s\left(5 k^{2}+9 k-8\right)} \bar{R}_{\max }^{(k+1, s)} .
\end{gathered}
$$


Thus, the assertion follows from (i) and the assumption.

This completes the proof.

q.e.d.

Theorem 12. Let $M^{n}$ be an $n(\geq 4)$-dimensional compact submanifold in an N-dimensional Riemannian manifold $\bar{M}^{N}$. Assume that $M$ satisfies one of the following conditions:

(i) $S<\frac{5 N}{6 s}\left[\overline{\operatorname{Ric}}_{\min }^{[s]}-\frac{s\left(5 N^{2}-5 N-12\right)}{5 N} \bar{K}_{\max }\right]+\frac{n^{2} H^{2}}{n-1}$;

(ii $S<\frac{N(N+1)}{3 s}\left[\overline{\operatorname{Ric}}_{\min }^{[s]}-\frac{s\left(N^{2}+2 N+3\right)}{N(N+1)} \overline{\operatorname{Ric}}_{\max }^{(N-2)}\right]+\frac{n^{2} H^{2}}{n-1}$;

(iii) $S<\frac{s N-s+6}{3}\left[\bar{K}_{\min }-\frac{1}{s N-s+6} \overline{R i c}[s] \max \right]+\frac{n^{2} H^{2}}{n-1}$;

(iv) $S<\frac{5 N^{2}-11 N-6}{6}\left[\overline{R i c}_{\min }^{(N-2)}-\frac{N(5 N-16)}{s\left(5 N^{2}-11 N-6\right)} \overline{\operatorname{Ric}}_{\max }^{[s]}\right]+\frac{n^{2} H^{2}}{n-1}$,

for some integer $s \in[2, N]$. Then the normalized Ricci flow with initial metric $g_{0}$,

$$
\frac{\partial}{\partial t} g(t)=-2 R i c_{g(t)}+\frac{2}{n} r_{g(t)} g(t)
$$

exists for all time and converges to a constant curvature metric as $t \rightarrow \infty$. Moreover, $M$ is diffeomorphic to a spherical space form. In particular, if $M$ is simply connected, then $M$ is diffeomorphic to $S^{n}$.

Proof. (i) Taking $k=N-1$ in (ii) of Lemma 10, we get the conclusion.

(ii) Since

$$
\begin{aligned}
\bar{K}_{\min } & \geq \frac{1}{2}\left[\bar{R}-(N+1) \overline{\operatorname{Ric}}_{\max }^{(N-2)}\right] \\
& \geq \frac{1}{2}\left[\frac{N \overline{\operatorname{Ric}}_{\min }^{[s]}}{s}-(N+1) \overline{\operatorname{Ric}}_{\max }^{(N-2)}\right],
\end{aligned}
$$

we obtain

$$
\begin{aligned}
\bar{K}_{\max } & \leq \overline{R i c}_{\max }^{(N-2)}-(N-3) \bar{K}_{\min } \\
& \leq \frac{N^{2}-2 N-1}{2} \overline{R i c}_{\max }^{(N-2)}-\frac{N(N-3)}{2 s} \overline{\operatorname{Ric}}_{\min }^{[s]}
\end{aligned}
$$

Suppose $\left\{e_{1}, e_{2}, e_{3}, e_{4}\right\}$ is an orthonormal four-frame and $\lambda \in \mathbb{R}$. Combining (2.1), (2.3), (4.8), (4.26), and (4.27), we obtain 


$$
\begin{aligned}
& R_{1313}+R_{2323}-\left|R_{1234}\right| \\
\geq & 2 \bar{K}_{\min }-\frac{2}{3}\left(\bar{K}_{\max }-\bar{K}_{\min }\right)+\sum_{\alpha}\left[h_{11}^{\alpha} h_{33}^{\alpha}+h_{22}^{\alpha} h_{33}^{\alpha}\right. \\
& \left.-\frac{3}{2}\left(h_{13}^{\alpha}\right)^{2}-\frac{3}{2}\left(h_{23}^{\alpha}\right)^{2}-\frac{1}{2}\left(h_{24}^{\alpha}\right)^{2}-\frac{1}{2}\left(h_{14}^{\alpha}\right)^{2}\right] \\
\geq & \frac{n^{2} H^{2}}{n-1}-S+\frac{4}{3}\left[\frac{N}{s} \overline{\operatorname{Ric}}_{\min }^{[s]}-(N+1) \overline{\operatorname{Ric}}_{\max }^{(N-2)}\right] \\
& -\frac{1}{3}\left[\left(N^{2}-2 N-1\right) \overline{R i c}_{\max }^{(N-2)}-\frac{N(N-3)}{s} \overline{R i c}_{\min }^{[s]}\right] \\
(4.28) \geq & \frac{N+1}{3}\left[\frac{N}{s} \overline{R i c}_{\min }^{[s]}-\frac{N^{2}+2 N+3}{N+1} \overline{R i c}_{\max }^{(N-2)}\right]+\frac{n^{2} H^{2}}{n-1}-S .
\end{aligned}
$$

A similar discussion implies that

$$
\begin{aligned}
& R_{1414}+R_{2424}-\left|R_{1234}\right| \\
\geq & \frac{N+1}{3}\left[\frac{N}{s} \overline{R i c}_{\min }^{[s]}-\frac{N^{2}+2 N+3}{N+1} \overline{R i c}_{\max }^{(N-2)}\right]+\frac{n^{2} H^{2}}{n-1}-S .
\end{aligned}
$$

From (4.28) and (4.29), we get

$$
\begin{array}{ll} 
& R_{1313}+\lambda^{2} R_{1414}+R_{2323}+\lambda^{2} R_{2424}-2 \lambda R_{1234} \\
\geq & R_{1313}+R_{2323}-\left|R_{1234}\right|+\lambda^{2}\left(R_{1414}+R_{2424}-\left|R_{1234}\right|\right) \\
\geq & \left(1+\lambda^{2}\right)\left\{\frac{N+1}{3}\left[\frac{N}{s} \overline{R i c}_{\min }^{[s]}-\frac{N^{2}+2 N+3}{N+1} \overline{R i c}_{\max }^{(N-2)}\right]\right. \\
& \left.+\frac{n^{2} H^{2}}{n-1}-S\right\} .
\end{array}
$$

Hence we get the conclusion from (4.30), the assumption, and Theorem A.

(iii) It's seen from (2.9) that

$$
\bar{K}_{\max } \leq \frac{1}{2}\left[\overline{\operatorname{Ric}}_{\max }^{[s]}-(s N-s-2) \bar{K}_{\min }\right] .
$$

Suppose $\left\{e_{1}, e_{2}, e_{3}, e_{4}\right\}$ is an orthonormal four-frame and $\lambda \in \mathbb{R}$. By (2.1), (2.3), (4.8), and (4.31), we have

$$
\begin{aligned}
& R_{1313}+R_{2323}-\left|R_{1234}\right| \\
\geq & 2 \bar{K}_{\min }-\frac{2}{3}\left(\bar{K}_{\max }-\bar{K}_{\min }\right)+\sum_{\alpha}\left[h_{11}^{\alpha} h_{33}^{\alpha}+h_{22}^{\alpha} h_{33}^{\alpha}\right. \\
& \left.-\frac{3}{2}\left(h_{13}^{\alpha}\right)^{2}-\frac{3}{2}\left(h_{23}^{\alpha}\right)^{2}-\frac{1}{2}\left(h_{24}^{\alpha}\right)^{2}-\frac{1}{2}\left(h_{14}^{\alpha}\right)^{2}\right] \\
\geq & \frac{8}{3} \bar{K}_{\min }-\frac{1}{3}\left[\overline{R i c}_{\max }^{[s]}-(s N-s-2) \bar{K}_{\min }\right]+\frac{n^{2} H^{2}}{n-1}-S \\
\geq & \frac{1}{3}\left[(s N-s+6) \bar{K}_{\min }-\overline{R i c}_{\max }^{[s]}\right]+\frac{n^{2} H^{2}}{n-1}-S .
\end{aligned}
$$


By using a similar argument, we get

$$
\begin{aligned}
& R_{1414}+R_{2424}-\left|R_{1234}\right| \\
\geq & \frac{1}{3}\left[(s N-s+6) \bar{K}_{\min }-\overline{R i c}_{\max }^{[s]}\right]+\frac{n^{2} H^{2}}{n-1}-S .
\end{aligned}
$$

It follows from (4.32) and (4.33) that

$$
\begin{array}{ll} 
& R_{1313}+\lambda^{2} R_{1414}+R_{2323}+\lambda^{2} R_{2424}-2 \lambda R_{1234} \\
\geq & R_{1313}+R_{2323}-\left|R_{1234}\right|+\lambda^{2}\left(R_{1414}+R_{2424}-\left|R_{1234}\right|\right) \\
\geq & \left(1+\lambda^{2}\right)\left\{\frac{1}{3}\left[(s N-s+6) \bar{K}_{\min }-\overline{R i c}_{\max }^{[s]}\right]+\frac{n^{2} H^{2}}{n-1}-S\right\},
\end{array}
$$

which together with the assumption and Theorem A implies the conclusion.

(iv) The assertion follows from (ii) of Lemma 12 by taking $k=N-2$. This proves the theorem. q.e.d.

Theorem 13. Let $M$ be a 3-dimensional compact submanifold in an $N$-dimensional Riemannian manifold $\bar{M}^{N}$. Assume that $M$ satisfies one of the following conditions:

(i) $S<\frac{1}{2}\left[\bar{R}-\left(N^{2}-N-4\right) \bar{K}_{\max }\right]+\frac{9}{2} H^{2}$;

(ii) $S<\bar{R}-(N+1) \overline{\operatorname{Ric}}_{\max }^{(N-2)}+\frac{9}{2} H^{2}$;

(iii) $S<2 \bar{K}_{\text {min }}+\frac{9}{2} H^{2}$;

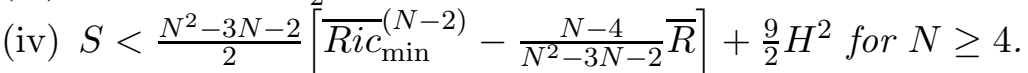

Then $M$ is diffeomorphic to a spherical space form. In particular, if $M$ is simply connected, then $M$ is diffeomorphic to $S^{n}$.

Proof. For any unit tangent vector $u \in U_{x} M$ at $x \in M$, we choose an orthonormal three-frame $\left\{e_{1}, e_{2}, e_{3}\right\}$ such that $e_{3}=u$.

(i) From (2.1), (4.8), and the assumption, we obtain

$$
\begin{aligned}
\operatorname{Ric}(u)= & R_{1313}+R_{2323} \\
= & \frac{1}{2}\left(\bar{R}-2 \sum_{A<B \neq 3} \bar{R}_{A B A B}\right) \\
& +\sum_{\alpha}\left[h_{11}^{\alpha} h_{33}^{\alpha}+h_{22}^{\alpha} h_{33}^{\alpha}-\left(h_{13}^{\alpha}\right)^{2}-\left(h_{23}^{\alpha}\right)^{2}\right] \\
\geq & \frac{1}{2}\left[\bar{R}-\left(N^{2}-N-4\right) \bar{K}_{\max }\right] \\
& +\sum_{\alpha}\left[\sum_{i \neq j}\left(h_{i j}^{\alpha}\right)^{2}+\frac{\left(\sum_{i=1}^{3} h_{i i}^{\alpha}\right)^{2}}{2}-S_{\alpha}-\left(h_{13}^{\alpha}\right)^{2}-\left(h_{23}^{\alpha}\right)^{2}\right] \\
\geq & \frac{1}{2}\left[\bar{R}-\left(N^{2}-N-4\right) \bar{K}_{\max }\right]+\frac{9}{2} H^{2}-S \\
> & 0 .
\end{aligned}
$$


(ii) It follows from (2.8) that

$$
\bar{K}_{\min } \geq \frac{1}{2}\left[\bar{R}-(N+1) \overline{\operatorname{Ric}}_{\max }^{(N-2)}\right],
$$

which together with $(2.1),(4.8)$, and the assumption implies

$$
\begin{aligned}
\operatorname{Ric}(u) & =R_{1313}+R_{2323} \\
& \geq 2 \bar{K}_{\min }+\sum_{\alpha}\left[h_{11}^{\alpha} h_{33}^{\alpha}+h_{22}^{\alpha} h_{33}^{\alpha}-\left(h_{13}^{\alpha}\right)^{2}-\left(h_{23}^{\alpha}\right)^{2}\right] \\
& \geq \bar{R}-(N+1) \overline{\operatorname{Ric}}_{\max }^{(N-2)}+\frac{n^{2} H^{2}}{n-1}-S \\
& >0 .
\end{aligned}
$$

(iii) From (2.1), (4.8), and the assumption, we obtain

$$
\begin{aligned}
\operatorname{Ric}(u) & =R_{1313}+R_{2323} \\
& \geq 2 \bar{K}_{\min }+\sum_{\alpha}\left[h_{11}^{\alpha} h_{33}^{\alpha}+h_{22}^{\alpha} h_{33}^{\alpha}-\left(h_{13}^{\alpha}\right)^{2}-\left(h_{23}^{\alpha}\right)^{2}\right] \\
& \geq 2 \bar{K}_{\min }+\frac{n^{2} H^{2}}{n-1}-S \\
& >0 .
\end{aligned}
$$

(iv) It's seen from (2.8) that

$$
\bar{K}_{\max } \leq \frac{1}{2}\left[\bar{R}-(N+1) \overline{R i c}_{\min }^{(N-2)}\right] .
$$

This together with (2.1), (4.8), and the assumption implies that

$$
\begin{aligned}
\operatorname{Ric}(u)= & R_{1313}+R_{2323} \\
\geq & \overline{R i c}_{\min }^{(N-2)}-(N-4) \bar{K}_{\max } \\
& +\sum_{\alpha}\left[h_{11}^{\alpha} h_{33}^{\alpha}+h_{22}^{\alpha} h_{33}^{\alpha}-\left(h_{13}^{\alpha}\right)^{2}-\left(h_{23}^{\alpha}\right)^{2}\right] \\
\geq & \overline{R i c}_{\min }^{(N-2)}-\frac{N-4}{2}\left[\bar{R}-(N+1) \overline{R i c}_{\min }^{(N-2)}\right]+\frac{n^{2} H^{2}}{n-1}-S \\
\geq & \frac{N^{2}-3 N-2}{2}\left[\overline{R i c}_{\min }^{(N-2)}-\frac{N-4}{N^{2}-3 N-2} \bar{R}\right]+\frac{n^{2} H^{2}}{n-1}-S \\
> & 0 .
\end{aligned}
$$

The assertion follows from (4.35), (4.37), (4.38), (4.40), and Hamilton's theorem [23]. This completes the proof of Theorem $13 . \quad$ q.e.d.

Theorem 14. Let $M^{n}$ be an $n(\geq 4)$-dimensional compact submanifold in an N-dimensional Riemannian manifold $\bar{M}^{N}$. Assume that $M$ satisfies one of the following conditions:

(i) $S<\frac{5}{6} N(N-1)\left(\bar{R}_{0}-\sigma_{N} \bar{K}_{\max }\right)+\frac{n^{2} H^{2}}{n-1}$,

(ii) $S<\frac{N\left(N^{2}-1\right)}{3(N-2)}\left[(N-2) \bar{R}_{0}-\mu_{N} \overline{R i c}_{\max }^{(N-2)}\right]+\frac{n^{2} H^{2}}{n-1}$, 
(iii) $S<\frac{N^{2}-N+6}{3}\left(\bar{K}_{\min }-\eta_{N} \bar{R}_{0}\right)+\frac{n^{2} H^{2}}{n-1}$,

(iv) $S<\frac{5 N^{2}-11 N-6}{6}\left[\overline{\operatorname{Ric}}_{\min }^{(N-2)}-\tau_{N}(N-2) \bar{R}_{0}\right]+\frac{n^{2} H^{2}}{n-1}$,

where $\sigma_{N}, \mu_{N}, \eta_{N}$, and $\tau_{N}$ are defined as in Theorems 1 and 2. Then $M$ is diffeomorphic to a spherical space form. In particular, if $M$ is simply connected, then $M$ is diffeomorphic to $S^{n}$.

Proof. By taking $k=N-1$ in (i) of Lemma 10, $s=N$ in (ii) and (iii) of Theorem 12, and $k=N-2$ in (i) of Lemma 12, respectively, we conclude that $M$ is diffeomorphic to a spherical space form. In particular, if $M$ is simply connected, then $M$ is diffeomorphic to $S^{n}$. This proves the theorem.

q.e.d.

\section{Submanifolds with weakly pinched curvatures}

In this section, we improve the differentiable sphere theorems [51] for submanifolds with strictly pinched curvatures and obtain a classification theorem for submanifolds with weakly pinched curvatures.

Proof of Theorem 3. If $n \geq 4$, suppose $\left\{e_{1}, e_{2}, e_{3}, e_{4}\right\}$ is an orthonormal four-frame and $\lambda \in \mathbb{R}$. From (2.1), (2.3), and (4.8), we have

$$
\begin{aligned}
& R_{1313}+\lambda^{2} R_{1414}+\mu^{2} R_{2323}+\lambda^{2} \mu^{2} R_{2424}-2 \lambda \mu R_{1234} \\
\geq & \left(1+\lambda^{2}+\mu^{2}+\lambda^{2} \mu^{2}\right) \bar{K}_{\min }-\frac{4|\lambda \mu|}{3}\left(\bar{K}_{\max }-\bar{K}_{\min }\right) \\
& +\sum_{\alpha}\left\{h_{11}^{\alpha} h_{33}^{\alpha}-\left(h_{13}^{\alpha}\right)^{2}+\mu^{2} \lambda^{2}\left[h_{22}^{\alpha} h_{44}^{\alpha}-\left(h_{24}^{\alpha}\right)^{2}\right]+2 \mu \lambda h_{14}^{\alpha} h_{23}^{\alpha}\right. \\
& \left.+\mu^{2}\left[h_{22}^{\alpha} h_{33}^{\alpha}-\left(h_{23}^{\alpha}\right)^{2}\right]+\lambda^{2}\left[h_{11}^{\alpha} h_{44}^{\alpha}-\left(h_{14}^{\alpha}\right)^{2}\right]-2 \mu \lambda h_{13}^{\alpha} h_{24}^{\alpha}\right\} \\
\geq & \left(1+\lambda^{2}+\mu^{2}+\lambda^{2} \mu^{2}\right) \frac{\left(4 \bar{K}_{\min }-\bar{K}_{\max }\right)}{3} \\
& +\sum_{\alpha}\left\{\sum_{i<j}\left(h_{i j}^{\alpha}\right)^{2}+\frac{\left(\sum_{i=1}^{n} h_{i i}^{\alpha}\right)^{2}}{2(n-1)}-\frac{S_{\alpha}}{2}-\left(h_{13}^{\alpha}\right)^{2}-\left(h_{14}^{\alpha}\right)^{2}-\left(h_{23}^{\alpha}\right)^{2}\right. \\
& +\mu^{2} \lambda^{2}\left[\sum_{i<j}\left(h_{i j}^{\alpha}\right)^{2}+\frac{\left(\sum_{i=1}^{n} h_{i i}^{\alpha}\right)^{2}}{2(n-1)}-\frac{S_{\alpha}}{2}-\left(h_{24}^{\alpha}\right)^{2}-\left(h_{14}^{\alpha}\right)^{2}-\left(h_{23}^{\alpha}\right)^{2}\right] \\
& +\mu^{2}\left[\sum_{i<j}\left(h_{i j}^{\alpha}\right)^{2}+\frac{\left(\sum_{i=1}^{n} h_{i i}^{\alpha}\right)^{2}}{2(n-1)}-\frac{S_{\alpha}}{2}-\left(h_{23}^{\alpha}\right)^{2}-\left(h_{13}^{\alpha}\right)^{2}-\left(h_{24}^{\alpha}\right)^{2}\right] \\
& \left.+\lambda^{2}\left[\sum_{i<j}\left(h_{i j}^{\alpha}\right)^{2}+\frac{\left(\sum_{i=1}^{n} h_{i i}^{\alpha}\right)^{2}}{2(n-1)}-\frac{S_{\alpha}}{2}-\left(h_{14}^{\alpha}\right)^{2}-\left(h_{13}^{\alpha}\right)^{2}-\left(h_{24}^{\alpha}\right)^{2}\right]\right\} \\
\geq \quad & \left(1+\mu^{2}+\lambda^{2}+\lambda^{2} \mu^{2}\right)\left[\frac{\left(4 \bar{K}_{\min }-\bar{K}_{\max }\right)}{3}\right. \\
(5.1)+ & \left.\frac{n^{2} H^{2}}{2(n-1)}-\frac{S}{2}\right] .
\end{aligned}
$$


From (5.1) and the assumption that $S \leq \frac{8}{3}\left(\bar{K}_{\min }-\frac{1}{4} \bar{K}_{\max }\right)+\frac{n^{2} H^{2}}{n-1}$, we have

$$
R_{1313}+\lambda^{2} R_{1414}+\mu^{2} R_{2323}+\lambda^{2} \mu^{2} R_{2424}-2 \lambda \mu R_{1234} \geq 0,
$$

i.e., $M \times \mathbb{R}^{2}$ has nonnegative isotropic curvature.

On the other hand, it follows from (2.1), (2.3), and (4.8) that

$$
\begin{aligned}
& R_{1313}+R_{2323}-\left|R_{1234}\right| \\
\geq & 2 \bar{K}_{\min }-\frac{2}{3}\left(\bar{K}_{\max }-\bar{K}_{\min }\right)+\sum_{\alpha}\left[h_{11}^{\alpha} h_{33}^{\alpha}+h_{22}^{\alpha} h_{33}^{\alpha}\right. \\
& \left.-\frac{3}{2}\left(h_{13}^{\alpha}\right)^{2}-\frac{3}{2}\left(a_{23}^{\alpha}\right)^{2}-\frac{1}{2}\left(h_{24}^{\alpha}\right)^{2}-\frac{1}{2}\left(h_{14}^{\alpha}\right)^{2}\right] \\
\geq & \frac{8}{3}\left(\bar{K}_{\text {min }}-\frac{1}{4} \bar{K}_{\max }\right)+\frac{n^{2} H^{2}}{n-1}-S .
\end{aligned}
$$

The equalities in (5.3) hold only if

$$
h_{i j}^{\alpha}=h_{33}^{\alpha}=0 \text {, for all distinct } i, j \text { and any } \alpha,
$$

and

$$
h_{i i}^{\alpha}=h_{j j}^{\alpha}, \text { for } i, j \neq 3 \text { and any } \alpha,
$$

$$
S=\frac{n^{2} H^{2}}{n-1} .
$$

It follows from (5.3), (5.5), and the assumption that the Ricci curvature of $M$ is quasi-positive. This together with Aubin's theorem [2] implies that $M$ admits a metric with positive Ricci curvature.

By a similar discussion, we have

$$
R_{1414}+R_{2424}-\left|R_{1234}\right| \geq \frac{8}{3}\left(\bar{K}_{\min }-\frac{1}{4} \bar{K}_{\max }\right)+\frac{n^{2} H^{2}}{n-1}-S .
$$

The equality in (5.6) holds only if

$$
h_{i j}^{\alpha}=h_{44}^{\alpha}=0 \text {, for all distinct } i, j \text { and any } \alpha,
$$

and

$$
h_{i i}^{\alpha}=h_{j j}^{\alpha}, \text { for } i, j \neq 4 \text { and any } \alpha .
$$

From (5.3), (5.5), and (5.6), we have

$$
\begin{aligned}
& R_{1313}+R_{1414}+R_{2323}+R_{2424}-2 R_{1234} \\
= & \left(R_{1313}+R_{2323}-\left|R_{1234}\right|\right)+\left(R_{1414}+R_{2424}-\left|R_{1234}\right|\right) \\
\geq & 2\left[\frac{8}{3}\left(\bar{K}_{\min }-\frac{1}{4} \bar{K}_{\max }\right)+\frac{n^{2} H^{2}}{n-1}-S\right],
\end{aligned}
$$

and the equality holds only if $S=\frac{n^{2} H^{2}}{n-1}$. This together with the assumption and Lemma 3 implies that $M$ admits a metric with positive 
isotropic curvature. By Lemma 4, we conclude that $M$ is diffeomorphic to a spherical space form. In particular, if $M$ is simply connected, then $M$ is diffeomorphic to $S^{n}$.

If $n=3$, for any unit tangent vector $u \in U_{x} M$ at $x \in M$, we choose an orthonormal three-frame $\left\{e_{1}, e_{2}, e_{3}\right\}$ such that $e_{3}=u$. From (4.8) and (4.38), we obtain

$$
\operatorname{Ric}(u)=R_{1313}+R_{2323} \geq 2 \bar{K}_{\min }+\frac{n^{2} H^{2}}{n-1}-S,
$$

and the equality holds only if $S=\frac{n^{2} H^{2}}{n-1}$. Then from the assumption we know that $M$ has quasi-positive Ricci curvature. Hence $M$ admits a metric with positive Ricci curvature by Aubin's theorem [2]. This together with Hamilton's theorem [23] implies that $M$ is diffeomorphic to a spherical space form. In particular, if $M$ is simply connected, then $M$ is diffeomorphic to $S^{n}$.

This completes the proof of Theorem 3 .

Corollary 2. Let $M^{n}$ be an $n(\geq 3)$-dimensional complete submanifold in an $N$-dimensional Riemannian manifold $\bar{M}^{N}$. If $S \leq \frac{8}{3}\left(\bar{K}_{\min }-\right.$ $\left.\frac{1}{4} \bar{K}_{\max }\right)+\frac{n^{2} H^{2}}{n-1}$ and the strict inequality holds for some point $x_{0} \in M$, then $M$ is diffeomorphic to a spherical space form or $\mathbb{R}^{n}$. In particular, if $M$ is simply connected, then $M$ is diffeomorphic to $S^{n}$ or $\mathbb{R}^{n}$.

Proof. From the assumption and Lemma 4.1 in [51], we know that $M$ has quasi-positive sectional curvature. When $M$ is noncompact, it follows from the Cheeger-Gromoll-Meyer-Perelman soul theorem $[\mathbf{1 4}, \mathbf{1 8}$, 33 that $M$ is diffeomorphic to $\mathbb{R}^{n}$. When $M$ is compact, the assertion follows from Theorem 3. This proves the corollary.

q.e.d.

For submanifolds in a sphere, we have the following theorem.

Theorem 15. Let $M^{n}$ be an $n$-dimensional compact submanifold in the unit sphere $S^{N}$. Assume that

$$
S \leq 2+\frac{n^{2} H^{2}}{n-1}
$$

We have the following possibilities:

(i) If $n=2$, then either $M$ is diffeomorphic to $S^{2}, \mathbb{R} P^{2}$, or $M$ is flat.

(ii) If $n=3$, then $M$ is diffeomorphic to a spherical space form.

(iii) If $n \geq 4$, then $M$ is diffeomorphic to $S^{n}$.

Proof. If $n=2$, it's seen from the Gauss equation that $2 K_{M}=2+$ $4 H^{2}-S$. This together with the assumption and the Gauss-Bonnet theorem implies the conclusion. 
If $n=3$, we see from Proposition 2.1 in [45] that

$$
\begin{aligned}
\operatorname{Ric}(u) \geq & \frac{2}{3}\left[3+6 H^{2}-S-\frac{3}{\sqrt{6}} H\left(S-3 H^{2}\right)^{1 / 2}\right] \\
= & \frac{2}{3}\left[\left(3+\frac{27}{4} H^{2}-\frac{3}{2} S\right)+\frac{3}{4} H^{2}\right. \\
& \left.+\frac{1}{2}\left(S-3 H^{2}\right)-\frac{3}{\sqrt{6}} H\left(S-3 H^{2}\right)^{1 / 2}\right] \\
\geq & 2+\frac{9}{2} H^{2}-S
\end{aligned}
$$

holds for any unit vector $u \in T_{x} M$ at each point $x \in M$, and the last inequality becomes an equality only if $S=\frac{9}{2} H^{2}$. This together with the assumption implies $M$ has positive Ricci curvature. Hence the assertion follows from Hamilton's theorem [23].

If $n \geq 4$, from (4.8) we get

$$
\begin{aligned}
& \sum_{k=2}^{n}\left[2\left|h\left(e_{1}, e_{k}\right)\right|^{2}-\left\langle h\left(e_{1}, e_{1}\right), h\left(e_{k}, e_{k}\right)\right\rangle\right]-(n-1) \\
= & \sum_{\alpha} \sum_{k=2}^{n}\left[2\left(h_{1 k}^{\alpha}\right)^{2}-h_{11}^{\alpha} h_{k k}^{\alpha}\right]-(n-1) \\
\leq & \sum_{\alpha} \sum_{k=2}^{n}\left\{2\left(h_{1 k}^{\alpha}\right)^{2}-\frac{1}{2}\left[\sum_{i \neq j}\left(h_{i j}^{\alpha}\right)^{2}+\frac{\left(\sum_{i=1}^{n} h_{i i}^{\alpha}\right)^{2}}{n-1}-S_{\alpha}\right]\right\} \\
& -(n-1) \\
\leq & \frac{n-1}{2}\left(S-\frac{n^{2} H^{2}}{n-1}-2\right) .
\end{aligned}
$$

The equalities in (5.9) hold only if $S=\frac{n^{2} H^{2}}{n-1}$. From the assumption $S \leq 2+\frac{n^{2} H^{2}}{n-1}$, we obtain

$$
\sum_{k=2}^{n}\left[2\left|h\left(e_{1}, e_{k}\right)\right|^{2}-\left\langle h\left(e_{1}, e_{1}\right), h\left(e_{k}, e_{k}\right)\right\rangle\right]-(n-1)<0 .
$$

This together with Theorem 5 implies that $M$ is simply connected. By Theorem 3, we see that $M$ is diffeomorphic to $S^{n}$. This proves Theorem 15.

q.e.d.

Moreover, we get the following classification for complete submanifolds in a Euclidean space.

Theorem 16. Let $M^{n}$ be an $n$-dimensional oriented complete submanifold in the Euclidean space $\mathbb{R}^{N}$. Assume that

$$
S \leq \frac{n^{2} H^{2}}{n-1}, \quad H \neq 0
$$

We have the following possibilities: 
(i) If $n=2$, then either $M$ is diffeomorphic to $S^{2}, \mathbb{R}^{2}$, or $M$ is flat.

(ii) If $n=3$, then $M$ is either diffeomorphic to a spherical space form, $\mathbb{R}^{3}$, or isometric to $S^{2}\left(r_{0}\right) \times \mathbb{R}$.

(iii) If $n \geq 4$, then $M$ is either diffeomorphic to $S^{n}, \mathbb{R}^{n}$, or locally isometric to $S^{n-1}(r) \times \mathbb{R}$.

Proof. It follows from the assumption and Lemma 1 that $K_{M} \geq 0$.

(i) Suppose that $M$ is compact. If $n=2$, it is seen from the GaussBonnet theorem that $M$ is diffeomorphic to $S^{2}$ or $M$ is flat.

If $n=3$, we know that $\operatorname{Ric}_{M} \geq 0$. This together with Hamilton's theorem [23] and Lemma 1 implies that $M$ is diffeomorphic to a spherical space form, or $H$ is a constant and $M$ is isometric to $S^{2}\left(\frac{2}{3 H}\right) \times \mathbb{R}$. Since $M$ is compact, the latter case is ruled out.

If $n \geq 4$, from the assumption $S \leq \frac{n^{2} H^{2}}{n-1}$ and Lemma 1, we know $\operatorname{Ric}_{M} \geq 0$. We claim that $M$ admits a metric with positive Ricci curvature. Otherwise, it's seen from Aubin's theorem [2] that for each point $x$ in $M$, there exists a unit vector $u$ in $T_{x} M$ such that $\operatorname{Ric}(u)=0$. By Lemma 1, we know that $H$ is constant and $M$ is isometric to $S^{n-1}\left(\frac{n-1}{n H}\right) \times \mathbb{R}$, which is noncompact. This contradicts the compactness of $M$. By the Bonnet-Myers theorem, the fundamental group $\pi_{1}(M)$ is finite. Moreover, from the assumption, we know that $S<\frac{n^{2} H^{2}}{n-2}$. It's seen from Lemma 2 that $H_{q}(M ; \mathbb{Z})=0$ for all $1<q<n-1$. Then we get from the universal coefficient theorem that $H^{n-1}(M ; \mathbb{Z})$ has no torsion, and hence neither does $H_{1}(M ; \mathbb{Z})$ by the Poincaré duality. This together with the fact that $\pi_{1}(M)$ is finite implies $H_{1}(M ; \mathbb{Z})=0$. Therefore we have $H_{n-1}(M ; \mathbb{Z})=0$. Denote by $\widetilde{M}$ the universal Riemannian covering of $M$. We may consider $\widetilde{M}$ to be a Riemannian submanifold of $\mathbb{R}^{N}$, and hence $\widetilde{M}$ is a homology sphere. Since $\widetilde{M}$ is simply connected, it is a topological sphere, which together with a result of Sjerve [46] implies that $M$ is simply connected.

On the other hand, from (5.1) and the assumption, we know that $M \times \mathbb{R}^{2}$ has nonnegative isotropic curvature. Moreover, it follows from (5.4), (5.7), and the assumption $H \neq 0$ that the equalities in (5.3) and (5.6) cannot hold simultaneously. Hence we see from (5.8) and the assumption that $M$ has positive isotropic curvature. It follows from Lemma 4 that $M$ is diffeomorphic to a spherical space form. Since $M$ is simply connected, $M$ is diffeomorphic to $S^{n}$.

(ii) Suppose $M$ is noncompact. If $n=2$, it follows from the CheegerGromoll-Meyer-Perelman soul theorem that $M$ is diffeomorphic to $\mathbb{R}^{2}$ or $M$ is flat.

If $n=3$, a theorem due to Schoen-Yau [38] and Zhu [57] states that if the Ricci curvature of $M$ is quasi-positive, then $M$ is diffeomorphic 
to $\mathbb{R}^{3}$. This together with Lemma 1 implies $M$ is diffeomorphic to $\mathbb{R}^{3}$ or isometric to $S^{2}\left(r_{0}\right) \times \mathbb{R}$.

If $n \geq 4$, we consider the following two cases:

Case I. $K_{M} \geq 0$ and $K(\pi)>0$ for any 2-plane $\pi \subset T_{x_{0}} M$ at some point $x_{0} \in M$. By the Cheeger-Gromoll-Meyer-Perelman soul theorem, we know that $M$ is diffeomorphic to $\mathbb{R}^{n}$.

Case II. For each point $x \in M$ there exists some 2-plane $\pi \subset T_{x} M$ such that $K(\pi)=0$. In this case, we get $S \equiv \frac{n^{2} H^{2}}{n-1}$. Moreover, it follows from Lemma 1 and a result due to Ozgur (see [31], Theorem 4.1) that $M$ is conformally flat. This together with a theorem due to Carron [13] implies that $M$ is diffeomorphic to $\mathbb{R}^{n}$ or locally isometric to $S^{n-1}(r) \times \mathbb{R}$.

This completes the proof of Theorem 16.

q.e.d.

Proof of Theorem 4. Combining Theorems 15 and 16 for $n \geq 4$, we complete the proof.

q.e.d.

Corollary 3. Let $M^{n}$ be an $n(\geq 4)$-dimensional oriented complete submanifold in an $N$-dimensional simply connected space form $F^{N}(c)$ with $c \geq 0$. Denote by $\operatorname{Ric}^{[s]}(\cdot)$ the s-th weak Ricci curvature of $M$. Assume that

$$
R i c_{\min }^{[s]} \geq \frac{s(n+1)(n-2) c}{n}+\frac{s n(n-2)}{n-1} H^{2}
$$

for some integer $s \in[1, n-1]$, where $c+H^{2}>0$. We have

(i) If $c=0$, then $M$ is either diffeomorphic to $S^{n}, \mathbb{R}^{n}$, or locally isometric to $S^{n-1}(r) \times \mathbb{R}$.

(ii) If $M$ is compact, then $M$ is diffeomorphic to $S^{n}$.

Proof. For $R \geq \frac{n}{s} R i c_{\mathrm{min}}^{[s]}$, the assertion follows from Theorem 4. q.e.d.

\section{References}

[1] B. Andrews \& C. Baker, Mean curvature flow of pinched submanifolds to spheres, J. Differential Geom. 85 (2010) 357-396, MR 2739807, Zbl 05842818.

[2] T. Aubin, Metriques Riemanniennes et courbure, J. Differential Geom. 4 (1970) 385-424, MR 0279731, Zbl 0212.54102.

[3] M. Berger, Riemannian geometry during the second half of the twentieth century, University Lecture Series, Vol. 17, American Mathematical Society, Providence, RI, 2000, MR 1729907, Zbl 0944.53001.

[4] C. Böhm \& B. Wilking, Manifolds with positive curvature operators are space forms, Ann. of Math. 167 (2008) 1079-1097, MR 2415394, Zbl 1185.53073.

[5] S. Brendle, A general convergence result for the Ricci flow in higher dimensions, Duke Math. J. 145 (2008) 585-601, MR 2462114, Zbl 1161.53052.

[6] S. Brendle, Ricci flow and the sphere theorem, Graduate Studies in Mathematics, Vol. 111, American Mathematical Society, 2010, MR 2583938, Zbl 1196.53001.

[7] S. Brendle, Einstein manifolds with nonnegative isotropic curvature are locally symmetric, Duke Math. J. 151 (2010) 1-21, MR 2573825, Zbl 1189.53042. 
[8] S. Brendle \& R. Schoen, Sphere theorems in geometry, Surveys in Differential Geometry, Vol. 13, 2009, 49-84, MR 2743447, Zbl 1184.53037.

[9] S. Brendle \& R. Schoen, Manifolds with 1/4-pinched curvature are space forms, J. Amer. Math. Soc. 22 (2009) 287-307, MR 2449060, Zbl pre05859406.

[10] S. Brendle \& R. Schoen, Classification of manifolds with weakly 1/4-pinched curvatures, Acta Math. 200 (2008) 1-13, MR 2386107,

[11] S. Brendle \& R. Schoen, Riemannian manifolds of positive curvature, Proceedings of the International Congress of Mathematicians, Hyderabad, India, 2010, MR 2827901, Zbl 1231.53032.

[12] H. D. Cao, B. L. Chen \& X. P. Zhu, Recent developments on Hamilton's Ricci flow, Surveys in Differential Geometry, Vol. 12, 2008, 47-112, MR 2488948, Zbl 1157.53002 .

[13] G. Carron \& M. Herzlich, Conformally flat manifolds with nonnegative Ricci curvature, Compositio Math. 142 (2006) 798-810, MR 2231203, Zbl 1100.53034.

[14] J. Cheeger \& D. Gromoll, On the structure of complete manifolds of nonnegative curvature, Ann. of Math. 96 (1972) 413-443, MR 0309010, Zbl 0246.53049.

[15] J. Cheeger \& T.H. Colding, On the structure of spaces with Ricci curvature bounded below. I, J. Differential Geom. 46 (1997) 406-480, MR 1484888, Zbl 0902.53034 .

[16] J. Deprez, Semi-parallel hypersurfaces, Rend. Sem. Mat. Univ. Politec. Torino 44 (1986) 303-316, MR 0906021, Zbl 0616.53018.

[17] K.D. Elworthy \& S. Rosenberg, Homotopy and homology vanishing theorems and the stability of stochastic flows, Geom. Funct. Anal. 6 (1996) 51-78, MR 1371231, Zbl 0858.58053.

[18] D. Gromoll \& W.T. Meyer, On complete open manifolds of positive curvature, Ann. of Math. 90 (1969) 75-90, MR 0247590, Zbl 0191.19904.

[19] K. Grove \& K. Shiohama, A generalized sphere theorem, Ann. of Math. 106 (1977) 201-211, MR 0500705, Zbl 0341.53029.

[20] H.P. Fu \& H.W. Xu, Vanishing and topological sphere theorems for submanifolds in hyperbolic space, Intern. J. Math. 19 (2008) 811-822, MR 2437072, Zbl 1191.49045.

[21] S. Harish, Manifolds with nonnegative isotropic curvature, Comm. Anal. Geom. 17 (2009) 621-635, MR 2601346, Zbl 1197.53047.

[22] P. Hartman, Oscillation criteria for self-adjoint second-order differential systems and "principal sectional curvature," J. Differential Equations 34 (1979) 326-338, MR 0550049, Zbl 0443.34029.

[23] R. Hamilton, Three manifolds with positive Ricci curvature, J. Differential Geom. 17 (1982) 255-306, MR 0664497, Zbl 0504.53034.

[24] R. Hamilton, Four-manifolds with positive isotropic curvature, Comm. Anal. Geom. 5 (1997) 1-92, MR 1456308, Zbl 0892.53018.

[25] G. Huisken, Flow by mean curvature of convex surfaces into spheres, J. Differential Geom. 20 (1984) 237-266, MR 0772132, Zbl 0556.53001.

[26] G. Huisken, Deforming hypersurfaces of the sphere by their mean curvature, Math. Z. 195 (1987) 205-219, MR 0892052, Zbl 0626.53039.

[27] G. Huisken, Contracting convex hypersurfaces in Riemannian manifolds by their mean curvature, Invent. Math. 84 (1986) 463-480, MR 0837523, Zbl 0589.53058. 
[28] B. Lawson \& J. Simons, On stable currents and their application to global problems in real and complex geometry, Ann. of Math. 98 (1973) 427-450, MR 0324529, Zbl 0283.53049.

[29] M. Micallef \& J.D. Moore, Minimal two-spheres and the topology of manifolds with positive curvature on totally isotropic two-planes, Ann. of Math. 127 (1988), 199-227, MR 0924677, Zbl 0661.53027.

[30] M. Micallef \& M. Wang, Metrics with nonnegative isotropic curvature, Duke Math. J. 72 (1993) 649-672, MR 1253619, Zbl 0804.53058.

[31] C. Ozgur \& M.M. Tripathi, On submanifolds satisfying Chen's equality in a real space form, Arab. J. Sci. Eng., Sect. A, Sci. 33 (2008) 321-330, MR 2467194, Zbl 1185.53060.

[32] G. Perelman, Manifolds of positive Ricci curvature with almost maximal volume, J. Amer. Math. Soc. 7 (1994) 299-305, MR 1231690, Zbl 0799.53050.

[33] G. Perelman, Proof of the soul conjecture of Cheeger and Gromoll, J. Differential Geom. 40 (1994) 209-212, MR 1285534, Zbl 0818.53056.

[34] P. Petersen, On eigenvalue pinching in positive Ricci curvature, Invent. Math. 138 (1999) 1-21, MR 1714334, Zbl 0988.53011.

[35] P. Petersen \& T. Tao, Classification of almost quarter-pinched manifolds, Proc. Amer. Math. Soc. 137 (2009) 2437-2440, MR 2495279, Zbl 1168.53020.

[36] P. Petersen \& F. Wilhelm, An exotic sphere with positive sectional curvature, arXiv:math. DG/0805.0812.

[37] H.E. Rauch, A contribution to differential geometry in the large, Ann. of Math. 54 (1951) 38-55, MR 0042765, Zbl 0043.37202.

[38] R. Schoen \& S.T Yau, Complete three-dimensional manifolds with positive Ricci curvature and scalar curvature, Ann. Math. Studies. 102 (1982) 209-228, MR 0645740, Zbl 0481.53036.

[39] R. Schoen \& S.T. Yau, Lectures on differential geometry, Conference Proceedings and Lecture Notes in Geometry and Topology I, International Press, Cambridge, MA, 1994, MR 1333601, Zbl 0830.53001.

[40] Z.M. Shen, A sphere theorem for manifolds of positive Ricci curvature, Indiana Univ. Math. J. 38 (1989) 229-233, MR 0982579, Zbl 0678.53032.

[41] Z.M. Shen, On complete manifolds of nonnegative $k$-th Ricci curvature, Trans. Amer. Math. Soc. 338 (1993) 289-310, MR 1112548, Zbl 0783.53026.

[42] K. Shiohama, A sphere theorem for manifolds of positive Ricci curvature, Trans. Amer. Math. Soc. 275 (1983) 811-819, MR 0682734, Zbl 0518.53047.

[43] K. Shiohama, Recent developments in sphere theorems, Differential Geometry: Riemannian Geometry, Proc. Symp. Pure Math. Vol. 54, Part 3, Amer. Math. Soc., 1993, pp. 551-576, MR 1216646, Zbl 0844.53035.

[44] K. Shiohama, Sphere theorems, Handbook of Differential Geometry, Vol. 1, F.J.E. Dillen and L.C.A. Verstraelen (eds.), Elsevier Science B.V., Amsterdam, 2000, pp. 965-903, MR 1736859, Zbl 0968.53003.

[45] K. Shiohama \& H.W. Xu, The topological sphere theorem for complete submanifolds, Compositio Math. 107 (1997) 221-232, MR 1458750, Zbl 0905.53038.

[46] D. Sjerve, Homology spheres which are covered by spheres, J. London Math. Soc. 6 (1973) 333-336, MR 0310895, Zbl 0252.57003.

[47] H. Wu, Manifolds of partially positive curvature, Indiana Univ. Math. J. 36 (1987) 525-548, MR 0905609, Zbl 0639.53050. 
[48] Y.L. Xin, Application of integral currents to vanishing theorems, Scient. Sinica (A) 27 (1984) 233-241, MR 0763966, Zbl 0554.49021.

[49] H.W. Xu, Recent developments in differentiable sphere theorems, Fifth International Congress of Chinese Mathematicians. Part 1, 2, 415-430, AMS/IP Stud. Adv. Math., 51, pt. 1, 2, Amer. Math. Soc., Providence, RI, 2012, MR 2908084, Zbl 1247.53040.

[50] H.W. Xu \& J. R. Gu, The differentiable sphere theorem for manifolds with positive Ricci curvature, Proc. Amer. Math. Soc. 140 (2012) 1011-1021, MR 2869085, Zbl pre06024108.

[51] H.W. Xu \& J. R. Gu, An optimal differentiable sphere theorem for complete manifolds, Math. Res. Lett. 17 (2010) 1111-1124, MR 2729635, Zbl 1221.53068.

[52] H.W. Xu \& J. R. Gu, Rigidity of Einstein manifolds with positive scalar curvature, preprint, 2010.

[53] H.W. Xu \& E. T. Zhao, Topological and differentiable sphere theorems for complete submanifolds, Comm. Anal. Geom. 17 (2009) 565-585, MR 2550209, Zbl 1185.53033.

[54] S.T. Yau, Open problems in geometry, Differential Geometry: Partial Differential Equations on Manifolds, Proc. Symp. Pure Math. Vol. 54, Part 1, Amer. Math. Soc., 1993, pp. 1-28, MR 1216573, Zbl 0801.53001.

[55] S.T. Yau \& R. Schoen, Lectures on Differential Geometry, Higher Education Press, Beijing, 2004. (in Chinese)

[56] S.T. Yau \& F. Y. Zheng, Negatively $\frac{1}{4}$-pinched Riemannian metric on a compact Kähler manifold, Invent. Math. 103 (1991) 527-535, MR 1091617, Zbl 0792.53064 .

[57] S.H. Zhu, On open three manifolds of quasi-positive Ricci curvature, Proc. Amer. Math. Soc. 120 (1994) 569-572, MR 1166362, Zbl 0790.53042.

Center of Mathematical Sciences ZHEJIANG UNIVERSITY HANGZHOU 310027, CHINA

E-mail address: gujr@cms.zju.edu.cn

Center of Mathematical Sciences ZHEJIANG UNIVERSITY Hangzhou 310027, China E-mail address: xuhw@cms.zju.edu.cn 